CERN-PPE/95-151

September 21, 1995

\title{
Energy and Spatial Resolution of a Shashlik Calorimeter and a Silicon Preshower Detector
}

\section{RD36 Collaboration}

P. Aspell, S. Bates, Ph. Bloch, R. Grabit, P. Jarron, F. Lemeilleur, R. Loos, A. Marchioro, E. Rosso CERN, Geneva, SWITZERLAND

J. Badier, J. Bourotte, A. Busata, Ph. Busson, C. Charlot, L. Dobrzynski, O. Ferreira, Ch. Gregory, A. Karar, P. Manigot, R. Tanaka, J.Ch. Vanel

LPNHE, Ecole Polytechnique, Palaiseau, FRANCE

S. Bityukov, V. Obraztsov, A. Ostankov, Yu. Protopopov, V. Rykalin, P. Spiridonov, V. Soushkov, V. Vasil'chenko IHEP, Protvino, RUSSIA

E. Clayton, D. Miller, C. Seez, T.S. Virdee

Imperial College, London, UK

R. Djilkibaev, S. Gninenko, E. Guschin, Y. Musienko, V. Popov, A. Skasyrskaya, I. Semenyuk INR, Moscow, RUSSIA

I. Cheremukhin, A. Egorov*), I. Golutvin, Y. Kozlov*), P. Moissenz, S. Sergueev, A. Sidorov*), E. Zubarev, N. Zamiatin JINR, Dubna, RUSSIA

P. Bordalo, S. Ramos, J. Varela LIP, Lisboa, PORTUGAL

D.J.A. Cockerill, J. Connolly, L. Denton Rutherford Appleton Laboratory, Didcot, UK

N. Godinovic, I. Puljak, I. Soric

FESB, University of Split, Split, CROATIA

S.R. Chendvankar, S.K. Gupta, S.N. Ganguli, A. Gurtu, M. Maity, G. Majumder, K.Mazumdar, T. Moulik

EHEP Group, Tata Institute of Fundamental Research, Colaba, Bombay, INDIA

*) Elma Industry, RUSSIA 


\section{Abstract}

New projective prototypes of scintillator/lead sandwich type sampling calorimeter Shashlik with silicon preshower detector have been constructed and tested with electron beam at CERN-SPS. The energy resolution is measured to be $8.7 \% / \sqrt{E(\mathrm{GeV})}$ in stochastic term, $0.330 / E(\mathrm{GeV})$ in noise term and $0.5 \%$ in constant term. The angular resolution is better than $70 \mathrm{mrad} / \sqrt{E(\mathrm{GeV})}$.

(To be submitted to Nuclear Instruments and Methods) 
An electromagnetic calorimeter at LHC should be capable of detecting the neutral Higgs boson in intermediate mass range which disintegrates into two photons. An excellent energy resolution must be assured with a good rapidity coverage. An ECAL option for the CMS detector at LHC $[1,2]$ is a lead/scintillator sandwich sampling calorimeter read by wave-length-shifting (WLS) fibres, called 'Shashlik' [3, 4]. This type of calorimeter was first proposed by H. Fessler et al. [5], and then has been tested by a IHEP/INR group [6]. Such calorimeters have also been tested at HERA [7] and are being constructed at DESY, E865 at BNL and DELPHI [8] at CERN. There are also R\&D projects on Shashlik type calorimeter carried out by collaborations of SPAKEBAB [9] at CERN, HERA-B [10] at DESY, and PHENIX [11] at BNL.

At LHC, a good photon pointing capability as well as a good $\pi^{0}$ rejection to eliminate backgrounds are also required. With the Shashlik design, a preshower detector is essential to guarantee these capabilities.

During spring of 1994, 36 projective Shashlik towers were constructed with special attention paid to mechanical tolerances. These towers, fulfilling the geometrical constraints of CMS, have been exposed to electrons at the CERN SPS-H4 beam line during 1994 with and without a preshower detector in front. The aim is to achieve the performance goals of the Shashlik calorimeter for CMS, i.e. a stochastic term of $\simeq 9 \% / \sqrt{E}$, a constant term of $\simeq 1 \%$, an energy equivalent of electronic noise of $300 \mathrm{MeV}$, and an angular resolution of $\sigma_{\theta}=70 \mathrm{mrad} / \sqrt{E}$, where $E$ is in $\mathrm{GeV}$.

This paper is organized as follows. The calorimeter prototypes are described in Section 2. The experimental setup is described in Section 3. The correction for lateral non-uniformity of Shashlik is discussed in Section 4. The energy and position/angular resolutions are discussed in Sections 5 and 6, respectively.

\section{Shashlik and Preshower Detector}

\subsection{Shashlik prototype}

The Shashlik calorimeter is based on a technique for readout of the scintillation light of a lead/scintillator sampling calorimeter with WLS fibres perpendicular to the plates. The first prototype (so called Nonet) was of parallelipedical shape [12, 13, 14, 15]. A prototype of 16 projective towers has been constructed in 1993. The beam test results without the magnetic field have been reported in previous notes [16, 17]. The light yield of these towers has been measured to be 12 photons/MeV. These projective towers were also tested in 3 Tesla magnetic field with PIN photodiode readout [4, 18, 19]. A light output increase $(+11 \%$ at 3 Tesla) was measured and no significant deterioration on Shashlik and preshower detector performance has been observed.

During the spring 1994, 36 new Shashlik projective towers were constructed. These towers designed for CMS geometry had smaller lateral dimension than the older ones and the fibre density was also changed. The basic parameters of the new projective prototypes are summarized in Table 1. The elementary tiles (scintillator/Tyvek/lead) are assembled using four stainless steel foils (50 and/or $100 \mu \mathrm{m}$ thick) welded on a stainless steel front and back plate. The towers are wrapped either with aluminum or aluminized mylar. To keep separate the lead and scintillator plates, Tyvek of $100 \mu \mathrm{m}$ is inserted between the plates. Thirty six WLS fibres are used to convert the scintillation light. The WLS fibre ends are aluminized at the front surface of the Shashlik. At the other end the fibres are bundled and coupled to silicon PIN photodiodes. The light yield of these towers significantly increased to 20 photons/MeV due to several factors : better construction quality, use of multiclad 


\begin{tabular}{|l|c|}
\hline Number of towers & 36 \\
\hline Tower lateral size & $\begin{array}{c}42 \times 42 \mathrm{~mm}^{2} \text { (front) } \\
\end{array}$ \\
\hline Number of planes & $70 \times 50 \mathrm{~mm}^{2}$ (rear) \\
\hline Scintillator/Lead & $3.75 \pm 0.05 \mathrm{~mm} / 2 \pm 0.005 \mathrm{~mm}$ \\
\hline \hline Total radiation length & $25.7 X_{0}$ \\
\hline Radiation length & $16.2 \mathrm{~mm}$ \\
\hline Molière radius & $34 \mathrm{~mm}$ \\
\hline \hline Scintillator & Polystyrene $+0.5 \%$ POPOP $+2 \%$ para-terphenyl \\
\hline WLS fibre & Y11, $\phi 1.0 \mathrm{~mm}$ \\
\hline Number of fibres & 36 \\
\hline Interfibre distance & Aluminized \\
\hline Front fibre ends & Photodiode + Amplifier \\
\hline \hline Readout
\end{tabular}

Table 1: Parameters for the new Shashlik projective towers.

fibres and use of Tyvek for lead/scintillator separation.

\subsection{Preshower detector setup}

The preshower detector gives the energy deposited in the first silicon layer after $2.05 X_{0}(5 \mathrm{~mm} \mathrm{Al}+11.2 \mathrm{~mm} \mathrm{~Pb})$ and in the second layer after $3 X_{0}(+5.6 \mathrm{~mm} \mathrm{~Pb})$. Each layer ( $400 \mu \mathrm{m}$ thick) consisted of 4 wafers (each $6 \times 6 \mathrm{~cm}^{2}$ with 29 strips with $2 \mathrm{~mm}$ pitch). Each strip had an area of $1.2 \mathrm{~cm}^{2}$ which corresponds to a detector capacitance of $40 \mathrm{pF}$. The signals were readout by a 16-channel AMPLEX-SiCAL signal processor developed by ECP/MIP for LEP experiments [20]. The schematic view of the test setup is given in Fig. 1.

\section{$3 \quad$ Experimental Setup \\ 3.1 SPS-H4 beam line}

The trigger (so called 'wide beam') is generated by the coincidence of three scintillation counters, S1 $\left(3 \times 3 \times 0.2 \mathrm{~cm}^{3}\right)$, S2A $\left(2 \times 2 \times 0.2 \mathrm{~cm}^{3}\right)$ and S2B $\left(2 \times 2 \times 0.2 \mathrm{~cm}^{3}\right)$. A set of 4 drift chambers with 4 planes each is used for tracking. There are 2 chambers for the measurement of the horizontal coordinate $x$, and 2 chambers for the vertical coordinate $y$. The distance between the two doublets each giving the $x, y$ coordinate is $95 \mathrm{~cm}$. The intrinsic resolution per plane is $210 \mu \mathrm{m}$ [21]. The impact point accuracy at the front surface of Shashlik has been estimated to be $240 \mu \mathrm{m}$ for $80 \mathrm{GeV} / \mathrm{c}$ particle. It is largely dominated by the track extrapolation error since the distance between Shashlik and the drift chamber is long (2.8 $\mathrm{m}$ from the last chamber to Shashlik surface). The material in front of the calorimeter amounted to $\sim 10 \%$ of $X_{0}$.

Special care has been taken in controlling the beam, especially for the study of energy resolution, in order to maintain the beam momentum spread well below the constant term of the calorimeter. The collimator slit, so called $C_{3}$, determines the beam energy spread of the SPS-H4 beam line ( $\pm 3 \mathrm{~mm}$ corresponds to $\pm 0.1 \%$ dispersion). The $C_{3}$ value has been chosen to get enough beam flux, producing a beam energy spread of $\pm 0.4,0.3$, $0.1,0.1,0.1,0.1,0.2$ and $0.3 \%$ for $10,15,20,35,50,80,120$ and $150 \mathrm{GeV}$ electron beam, 
respectively. These values are quadratically subtracted from observed energy resolution when fitting the data.

We used a moving table which permitted motion in three directions, a horizontal translation, and rotation along two orthogonal angles $\theta$ and $\phi$. The distance between the pivot point of the moving table and the front surface of Shashlik has been $1.40 \mathrm{~m}$, corresponding to the radial position of ECAL from the interaction point in CMS.

The matrix of Shashlik towers is a square $6 \times 6$ matrix. All the towers have been tilted by 3 degrees in one direction while they have been kept projective in the other direction. The 4 central towers, later referred as Tower-15, 16, 21 and 22, have been exposed to electrons of various energies to study energy resolution. Tower-21 is a special one since the WLS fibres are also projective (thus the fibre density varies as a function of the depth of the shower). All other towers have a constant distance between fibres (parallel WLS fibre) which permits compensation between light collection efficiency (less efficient for deeper scintillator layers) and light loss due to WLS fibre attenuation. For the uniformity study, the whole area of the $6 \times 6$ matrix is scanned with 80 and $150 \mathrm{GeV}$ electron beams. Some data with muons $(225 \mathrm{GeV})$ have also been taken to study response to minimum ionizing particles (mip).

\subsection{Readout electronics}

Since a standard photomultiplier does not operate in a strong magnetic field (greater than 1 Tesla), the front-end readout system consisted of a silicon PIN photodiode followed by a pre-amplifier and a line driver. The general readout scheme is shown in Fig. 2. The signals are fed into the amplifier and shaper with a shaping time of 30 - $40 \mathrm{~ns}$. Then signals are transmitted through a $90 \mathrm{~m}$ twisted pair cable, and received with a post-amplifier which divides the signal into low and high gains. The ratio between low and high gain has been set to 10. The ADC's used are LeCroy FERA ADC's (11 bits). Due to the signal attenuation and the degradation after $90 \mathrm{~m}$ cable, the gate width for the ADC has been set to $160-200 \mathrm{~ns}$.

\subsubsection{PIN photodiode}

The scintillation light signals are detected with a silicon photodiode (Hamamatsu S3590-05) which has an area of $1 \times 1 \mathrm{~cm}^{2}$ (active area $9 \times 9 \mathrm{~mm}^{2}$ ) with a reverse bias voltage of 80 Volts to get full depletion. The PIN photodiode has a thickness of $500 \mu \mathrm{m}$ which corresponds to a detector capacitance of $27 \mathrm{pF}$ at full depletion bias voltage. The leakage current is small, below $8 \mathrm{nA}$. The quantum efficiency of this photodiode is $68 \%$ at the WLS fibre output $\lambda=550 \mathrm{~nm}$.

\subsubsection{Preamplifier, Shaper and Line-Driver}

In the PIN photodiode the electron-hole pairs directly produced by the scintillation light constitute the charge signal (typically only 10 - 13 electron-hole pairs created per $\mathrm{MeV}$ of energy loss in the Shashlik calorimeter). The use of a low-noise amplifier is therefore required. Also a fast shaping of signals is required to keep the noise from energy pileup small when running at high luminosities at the LHC. The equivalent noise charge (ENC) of the amplifier depends directly on the noise performance of the first transistor (junction FET) and on the detector characteristics. A JFET-KP341, characterised by high transconductance $(25 \mathrm{~mA} / \mathrm{V})$ and low input capacitance $(5 \mathrm{pF})$ has been chosen because of its good low-noise performance for the detector capacitance in the range $0-50 \mathrm{pF}$. A fast low-noise charge amplifier has been designed as a hybrid variant. The overall linear chain 
of the amplifier is shown in Fig. 3. The second stage provides additional amplification and cancellation of the signal tail that reduces the signal length to $30 \mathrm{~ns}$.

The typical delta current response of the amplifier is shown in Fig. 4 for two different detector capacitances. The design allows an increase of the shaping time using an external capacitance. The maximum negative output voltage swing of this amplifier is $2.5 \mathrm{~V}$. Its power consumption, determined essentially by the second stage is about $400 \mathrm{~mW}$ per channel. The linearity performance for a gate width of $50 \mathrm{~ns}$, is better than $\pm 1 \%$ in the range of $10^{3}-2 \times 10^{6}$ electrons at the input. The ENC of the hybrid amplifier has been measured for several capacitances up to $70 \mathrm{pF}$. The results are shown in Fig. 5. The measured overall gain of the amplifier is $5.4 \mathrm{mV} / \mathrm{fC}$. For Hamamatsu PIN photodiode (S3590-05) with detector capacitance of $C_{d}=27 \mathrm{pF}$, the equivalent noise charge (ENC) is 1300 electrons.

\section{Correction of Lateral Non-uniformity}

The lateral non-uniformity is mainly caused by the optical property of the Shashlik tower. Due to the choice of parallel fibres, light collection is less efficient at the edge of the tiles. The global lateral non-uniformity reaches the value of $-7 \%$ at the tower edge compared to the tower center, but this can be corrected globally by a 2 nd order polynomial [16]. In addition, if the particles hit the calorimeter at normal incidence, there are effects due to Cerenkov light, scintillation in fibre and shower leakage due to air gap between fibre and scintillator. We parametrize this local fibre effect with a cos-wave function. The total correction is therefore,

$$
f(E, B, \theta)=a_{1}\left\{1-a_{2}\left(x-x_{0}\right)^{2}\right\} \cdot\left\{1-a_{3} \cos \left\{\frac{2 \pi}{d}\left(x-x_{0}\right)\right\}\right\}
$$

where parameters $a_{i}$ represent :

$-a_{1}$ : calorimeter response at the center,

$-a_{2}$ : global non-uniformity correction coefficient,

$-a_{3}$ : local non-uniformity correction coefficient,

and the geometrical parameters $d$ and $x_{0}$ represent the inter-fibre distance and the tower center position, respectively.

For the correction, the beam chamber information $x$ is used. In principle, a calorimeter based impact point calculation should be used since tracking information will be absent for neutral particles. This can be easily done by replacing $x$ in the equation with $x=f^{-1}(A)$, where $A$ is an energy asymmetry variable given by,

$$
A=\frac{E_{L}-E_{R}}{E_{t o t}}
$$

where $E_{L}\left(E_{R}\right)$ is the energy in left (right) column in $3 \times 3$ cluster, and $E_{\text {tot }}$ is the total energy. $f$ is the S-curve function which gives the relation between $x$ and asymmetry. When the energy asymmetry variable is used, the correction functions are energy dependent (more precise for higher energies) and also position dependent (more precise at tower boundary region).

One example of the correction for lateral non-uniformity is given in Fig. 6. Similar results are obtained for Towers-15, 16 and 22 (parallel WLS fibres). For Tower-21 (projective WLS fibres), strong local peaks (not shown) at the fibre positions are observed.

Any non-uniformity contributes to the constant term in energy resolution. After correction for this non-uniformity the residual contribution is well below $0.2 \%$. 
For the study of energy resolution, events with 'wide beam' $\left(2 \times 2 \mathrm{~cm}^{2}\right)$ trigger are analysed and the lateral non-uniformity correction is applied to extract the intrinsic Shashlik energy resolution.

\subsection{Shashlik signal linearity}

A factor which may worsen the energy resolution is the non-linearity of the readout system. Although this non-linearity should cancel out in first order for the study of the energy resolution (dispersion divided by mean value), it could not be totally negligible. The linearity of the electronics chain has been checked with a test pulse injected into the $1.5 \mathrm{pF}$ capacitor in the preamplifier. Long square pulses were attenuated by HP VHF$355 \mathrm{C} / \mathrm{D}$ and then transmitted to signal splitter to deliver the signal to all channels. Data have been corrected for both low and high gain channels. The linearity of the Shashlik signal in $3 \times 3$ cluster normalized at $80 \mathrm{GeV}$ is shown in Fig. 7 for 4 different towers. After the correction, the residual non-linearity is less than $\pm 2 \%$.

\subsection{Preshower detector performance}

Typically an electronics noise equivalent to $0.15 \mathrm{mip}$ was measured, where one mip left $140 \mathrm{keV}$ in each silicon layer. Uniformity and calibration study with $225 \mathrm{GeV}$ muons and electrons showed that the dispersion in response between strips in one plane is $\pm 3 \%$, thus no correction has been applied. The mean signal for electrons is shown in Fig. 8. A good agreement is observed between data and the predictions of a Monte Carlo simulation.

\subsection{Energy resolution of bare Shashlik}

The energy resolution of a calorimeter is generally parametrized as,

$$
\frac{\sigma_{E}}{E}=\frac{a}{\sqrt{E}} \oplus \frac{b}{E} \oplus c,
$$

where $a$ gives the stochastic term, $b$ the noise term, $c$ the constant term, and $E$ is the energy in $\mathrm{GeV}$.

The energy resolution is studied using energy summed over 9 towers with the beam incident over an area of $2 \times 2 \mathrm{~cm}^{2}$ in the center of the central tower. The lateral nonuniformity correction described above is applied. The reconstructed energy in the nine towers is shown in Fig. 9 and 10 for electrons 80 and $150 \mathrm{GeV}$, respectively. The tail on the high energy side is due to the "nuclear counter effect" (signal equivalent to $1.25 \mathrm{GeV}$ per minimum ionizing particle). To extract the intrinsic resolution, a fit to a Gaussian plus a Landau distribution has been performed. A summary of Shashlik energy resolution is given in Table 2 and shown in Fig. 11 for four towers, T-15,16,21 and 22. Tower-21 had a special geometry as the WLS fibres are projective. For the high energy data of this tower, we have observed a non-Gaussian tail due to Čerenkov light in the fibre and light loss due to the air gap between fibre and scintillator plates. We therefore have observed a slightly worse constant term for Tower-21. The average resolution of the calorimeter is,

$$
\frac{\sigma_{E}}{E}=\frac{8.1 \%}{\sqrt{E}} \oplus \frac{0.330}{E} \oplus 0.5 \%
$$

which is well within the design parameters for a sampling calorimeter in CMS $[1,2]$. 


\begin{tabular}{|l||c|c|c|}
\hline \multicolumn{1}{|l||}{ Tower } & \multicolumn{3}{c|}{ Energy Resolution } \\
\hline & Stochastic & Noise & Constant \\
\hline \hline Tower-15 & $7.45 \pm 0.35 \%$ & $\begin{array}{l}361 \pm 14 \mathrm{MeV} \\
(120 \mathrm{MeV} / \mathrm{ch})\end{array}$ & $0.49 \pm 0.04 \%$ \\
& & $\begin{array}{l}351 \pm 15 \mathrm{MeV} \\
(117 \mathrm{MeV} / \mathrm{ch})\end{array}$ & $0.54 \pm 0.04 \%$ \\
\hline Tower-16 & $8.10 \pm 0.35 \%$ & $0.72 \pm 0.03 \%$ \\
& & $\begin{array}{l}308 \pm 17 \mathrm{MeV} \\
(103 \mathrm{MeV} / \mathrm{ch})\end{array}$ & \\
\hline Tower-21 & $7.83 \pm 0.36 \%$ & $\begin{array}{l}307 \pm 17 \mathrm{MeV} \\
(102 \mathrm{MeV} / \mathrm{ch})\end{array}$ & $0.52 \pm 0.04 \%$ \\
\hline Tower-22 & $8.98 \pm 0.31 \%$ & $0.5 \%$ \\
\hline \hline Average & $8.1 \%$ & $330 \mathrm{MeV}$ & 0.5 \\
\hline \hline
\end{tabular}

Table 2: Summary of test beam results of new projective towers. The energy resolution parameters of bare Shashlik are shown.

Results of a Monte Carlo simulation with the exact setup are shown in Fig. 12 for zero or $30 \%$ of $X_{0}$ absorber material in front of the calorimeter. This Monte Carlo simulation included the saturation effect in the scintillator, and we have assumed a laterally infinite calorimeter. The light collection has also been simulated taking into account, light reflection efficiency inside scintillator, light attenuation in the WLS fibres, and quantum efficiency of silicon PIN photodiode. The sampling fluctuation dominates the stochastic term. With no material in front of the calorimeter, we obtain a $7.3 \%$ stochastic term. With $30 \%$ of $X_{0}$ material, the stochastic term still remains within $8 \%$. These values are in agreement with the value observed $(8.1 \%)$ for the test beam condition $\left(10 \% X_{0}\right.$ in front of calorimeter).

As for the constant term, several sources have been considered. They are: longitudinal non-uniformity (due to variations in scintillator light yield or in light collection), longitudinal shower leakage and residual lateral non-uniformity. The effect of the shower leakage from rear of the calorimeter has been simulated for high energy electrons $(200 \mathrm{GeV})$. In Fig. 13, the contribution to the energy resolution is shown as functions of the total calorimeter length and of the leaking energy. No sampling fluctuation and no noise term had been given in this simulation. Only Landau fluctuation (negligible) or shower leakage would contribute to the energy resolution. The energy resolution, therefore, should asymptotically reach $0 \%$ for a infinitely long calorimeter. For bare Shashlik with finite length $\left(25.7 X_{0}\right)$, one of the main source of the constant term is due to the shower leakage from backside, which contributes roughly $0.4 \%$ to the constant term. For Shashlik plus preshower detector configuration $\left(25.7+3.0 X_{0}\right)$, the contribution to the constant term is as small as $0.2 \%$.

\subsection{Energy resolution on a large area}

The energy resolution for an area covering 16 towers has been measured with 80 and $150 \mathrm{GeV}$ electrons. The results for $80 \mathrm{GeV}$ data are shown in Fig. 14 after full uniformity correction. The average energy resolution is $\sigma / E \simeq 1.2 \%$ in the tower centers $\left(2 \times 2 \mathrm{~cm}^{2}\right)$ and compatible with the results obtained with energy scan data described before. For the full tower surface $\left(4.7 \times 4.7 \mathrm{~cm}^{2}\right)$, the average energy resolution is $\simeq 1.3 \%$. The dispersion in the energy resolution for the 16 individual towers is small (of the order of $0.1 \%$ ). When 
all data are used $\left(20 \times 15 \mathrm{~cm}^{2}\right)$ the overall resolution $(1.29 \%)$ is also compatible with the one obtained for the individual towers. If one fixes the stochastic term to $8.1 \%$, all these data gives the constant term well below $1.0 \%$.

\subsection{Energy resolution of Shashlik + preshower detector}

Since the preshower detector system contains a $3 X_{0}$ absorber placed in front of the Shashlik calorimeter, a fraction of energy is lost and must be corrected for, using the signals observed in the two silicon planes. One would therefore expect to reconstruct the incident electron energy by the expression :

$$
E_{\text {beam }}=\alpha E_{p s 1}+\beta E_{p s 2}+E_{S h},
$$

where $E_{\text {beam }}$ is the incident beam energy and $E_{p s 1, p s 2}$ are the energies measured in the two silicon layers with suitable coefficients $\alpha$ and $\beta$. And $E_{S h}$ is the energy measured in the Shashlik calorimeter, using the calibration obtained without preshower detector. However, the estimate of the total energy is complicated by four additional effects.

\section{i) Change in sampling fraction}

The sampling fraction (the ratio of light in scintillator to the energy deposited in the preceding calorimeter absorber plate) varies while shower develops in the calorimeter. This is due to the soft particles (photons) which are abundant as electromagnetic shower develops. The calorimeter response to these low-energy photons is relatively suppressed compared to electrons/positrons [22]. The sampling fraction is higher at early shower development. As a consequence, the same energy in the calorimeter will produce different light output depending on the presence or absence of the preshower detector.

\section{ii) Longitudinal shower dependence}

The compensation between light collection and fibre attenuation depends on the longitudinal profile, which will be different when the preshower detector is inserted.

\section{iii) Front material of Shashlik}

Some of the low energy particles present in the shower after the preshower radiator are absorbed in the front material of the Shashlik tower. The results of a Monte Carlo simulation with GEANT 3.21 are shown in Fig. 15 for the complete geometry of the calorimeter system. The average energies deposited in each material for $40 \mathrm{GeV}$ electrons are summarized in Table 3. These materials include $10 \% X_{0}$ absorber in front of the setup, the preshower detector $\left(3 X_{0}\right)$, the front material inside the Shashlik tower $(5 \mathrm{~mm}$ plastic $+2 \mathrm{~mm}$ inox $+5 \mathrm{~mm}$ plastic). In the case of the bare Shashlik $\left(25.7 X_{0}\right)$, only $0.8 \%$ of the incident energy is lost due to shower leakage from front/backside, and to the material before Shashlik. When the preshower detector is inserted, the energy lost in the front material of the Shashlik tower is as large as that in the first preshower detector layer $\left(2 X_{0}\right)$. This effect is strongly energy dependent, and the fluctuation will give an additional contribution to the constant term in energy resolution. For future design, this front material has to be optimized to reduce the observed effect.

\section{iv) Non-linearity of readout system}

Finally, the non-linearity of the readout system will produce different energy response for a single incident electron and a degraded shower.

For all these reasons, it is not possible to determine in a absolute way the energy deposited in Shashlik calorimeter using the calibration obtained without preshower detector. To overcome this problem, we introduced a third parameter $\gamma$ applied to the Shashlik 


\begin{tabular}{|r||rr|rrr|}
\hline & $E_{\text {abso }}$ & $E_{p s}$ & $E_{\text {front }}$ & $E_{\text {lead }}$ & $E_{\text {scinti }}$ \\
\hline \hline without preshower & 0.02 & - & 0.02 & 33.72 & 5.95 \\
with preshower & 0.02 & 1.39 & 0.46 & 32.34 & 5.62 \\
\hline \hline
\end{tabular}

Table 3: The average deposit energies in absorber material, preshower detector and in Shashlik (front material, lead and scintillators) for $40 \mathrm{GeV}$ electrons. The unit is in GeV.

\begin{tabular}{|c|rrrrrr|}
\hline $\mathrm{E}_{\text {beam }}(\mathrm{GeV})$ & 10 & 20 & 35 & 50 & 80 & 120 \\
\hline \hline$\alpha \times 10^{3}$ & 15.4 & 15.5 & 15.0 & 15.0 & 16.0 & 17.1 \\
$\beta \times 10^{3}$ & 11.5 & 12.2 & 11.4 & 11.5 & 11.5 & 14.4 \\
\hline \hline
\end{tabular}

Table 4: Average values of parameters $\alpha$ and $\beta$ as a function of electron incident energy for Shashlik Tower-15 and 16. The parameters $\alpha$ and $\beta$ are expressed in GeV/MIP.

energy, and the energy resolution for Shashlik plus preshower detector system has been studied by minimizing,

$$
\chi^{2}=\sum_{\text {evt }}\left(E_{\text {beam }}-\alpha E_{p s 1}-\beta E_{p s 2}-\gamma E_{S h}\right)^{2} .
$$

Similar results could be obtained by fitting the anticorrelation curve between the preshower energy and the Shashlik energy, namely without knowing the beam energy. The summary of the measured values of the parameters $\alpha$ and $\beta$ are given in Table 4, and they were practically energy independent. The energies measured with this method are shown in Fig. 16 for $150 \mathrm{GeV}$ electrons. The spectrum fits very well a Gaussian. Because of the additional $3 X_{0}$ of the preshower detector, the non-Gaussian tail due to shower leakage or nuclear counter effect in silicon photodiode is absent.

The results on the energy resolution are shown in Fig. 17 and summarized in Table 5 for four towers T-15,16,21 and 22. The energy resolution of the bare calorimeter is fully recovered above $40 \mathrm{GeV}$. The average resolution is,

$$
\frac{\sigma_{E}}{E}=\frac{8.7 \%}{\sqrt{E}} \oplus \frac{0.330}{E} \oplus 0.5 \% .
$$

The stochastic term is slightly worse (by $0.6 \%$ ) than for Shashlik alone, but the same constant term has been observed. The noise term has been fixed to the value obtained for Shashlik alone because no additional noise contribution is expected from preshower detector system.

\section{$6 \quad$ Position and Angular Resolution}

\subsection{Position resolution of Shashlik and preshower detector}

To measure the Shashlik and preshower detector's position resolution, it is necessary to deconvolute the beam chamber resolution and the beam divergence. The later is the dominant effect, because the distance between the last chamber and the calorimeter has been $2.8 \mathrm{~m}$ in the 1994 SPS-H4 setup : to keep the track extrapolation error smaller than the preshower detector resolution $(\ll 300 \mu \mathrm{m})$, the beam divergence must be known with a precision better than $\sim 8 \times 10^{-5} \mathrm{rad}$. Unfortunately, the precision is limited to $2.2 \times 10^{-4} \mathrm{rad}$ due to the small distance $(\sim 1 \mathrm{~m})$ between the two sets of beam chamber. 


\begin{tabular}{|c||c|c|c|}
\hline \multicolumn{1}{|l||}{ Tower } & \multicolumn{3}{c|}{ Energy Resolution } \\
\hline & Stochastic & Noise & Constant \\
\hline \hline Tower-15 & $8.47 \pm 0.18 \%$ & $\begin{array}{c}361 \mathrm{MeV} \text { fix } \\
(120 \mathrm{MeV} / \mathrm{ch})\end{array}$ & $0.38 \pm 0.04 \%$ \\
\hline Tower-16 & $9.12 \pm 0.17 \%$ & $\begin{array}{c}351 \mathrm{MeV} \text { fix } \\
(117 \mathrm{MeV} / \mathrm{ch})\end{array}$ & $0.27 \pm 0.05 \%$ \\
\hline Tower-21 & $8.82 \pm 0.18 \%$ & $\begin{array}{c}308 \mathrm{MeV} \text { fix } \\
(103 \mathrm{MeV} / \mathrm{ch})\end{array}$ & $0.42 \pm 0.03 \%$ \\
\hline Tower-22 & $8.62 \pm 0.20 \%$ & $\begin{array}{c}307 \mathrm{MeV} \mathrm{fix} \\
(102 \mathrm{MeV} / \mathrm{ch})\end{array}$ & $0.80 \pm 0.02 \%$ \\
\hline \hline Average & $8.7 \%$ & $330 \mathrm{MeV}$ & $0.5 \%$ \\
\hline \hline
\end{tabular}

Table 5: Summary of the energy resolution of Shashlik plus preshower detector. The noise term has been fixed at that of bare Shashlik study.

\begin{tabular}{|c||c|c|}
\hline Energy $(\mathrm{GeV})$ & Real beam divergence & Effect on resolution \\
\hline \hline 120 & $0.4 \times 10^{-4}$ & $180 \mu \mathrm{m}$ \\
80 & $0.6 \times 10^{-4}$ & $250 \mu \mathrm{m}$ \\
35 & $1.0 \times 10^{-4}$ & $415 \mu \mathrm{m}$ \\
\hline \hline
\end{tabular}

Table 6: Beam divergence effect on preshower detector resolution for various energies.

To overcome the problem, two different approaches have been used. In the first method, the beam divergence is deduced from the known SPS-H4 beam properties, in particular from the value of the collimator slit $C_{2}$. In the second approach, we measure the weak - but non vanishing - correlation between the beam divergence and the difference between the preshower and beam coordinates. This correlation is compared with the same correlation obtained in a Monte Carlo simulation for which the beam divergence is the only variable parameter.

The two methods gave similar results. The beam divergence and its effect on resolution are listed in Table 6 . At $80 \mathrm{GeV}$, the preshower detector resolution in the $3 X_{0}$ plane is measured to be $313 \mu \mathrm{m}$ ( $403 \mu \mathrm{m}$ before subtraction) and is shown in Fig. 18.

For Shashlik, the position resolution is worse and therefore less sensitive to the beam divergence. The calibration of the Shashlik position $X_{S h}$ has been done by fitting the asymmetry measured with Shashlik towers as a function of $X_{\text {beam }}$ with a polynomial line or a modified hyperbolic tangent,

$$
X_{\text {beam }}=a+b \times A+c \times\{\tanh (d * A)\},
$$

where $\mathrm{A}$ is the asymmetry value, and a,b,c and d are free parameters. The Shashlik position resolution for the full front surface studied using $80 \mathrm{GeV}$ electrons is shown in Fig. 19. The resolution at the tower edge for the tilted case is as foreseen slightly worse than that for non-tilted case. Fig. 20 shows the position resolution of Shashlik as a function of the energy. It should be noted that the energy scan data for Shashlik tower corresponds to the center of this tower, i.e. $\pm 10 \mathrm{~mm}$ region. Also shown, for $80 \mathrm{GeV}$ electrons, is the resolution for an area covering the full Tower- 22 and adjacent half towers. The resolution 


\begin{tabular}{|l||r|r|r|r|r|r|}
\hline Energy (GeV) & 20 & 35 & 50 & 80 & 120 & 150 \\
\hline \hline$\Delta X$ (r.m.s.) (mm) & 2.00 & 1.65 & 1.30 & 1.12 & 0.90 & 0.86 \\
$<l>$ & 15.9 & 17.1 & 17.9 & 18.9 & 19.8 & 20.3 \\
$L(\mathrm{~mm})$ & 116 & 125 & 133 & 143 & 149 & 152 \\
$\Delta$ Angle (mrad) & 17.2 & 13.2 & 9.7 & 7.8 & 6.0 & 5.65 \\
\hline \hline
\end{tabular}

Table 7: Summary of position and angular resolution.

on the difference between the Shashlik and preshower detector coordinate $\Delta X=X_{S h}-X_{p s}$ is listed in Table 7. This difference is in principle free of the beam chamber error.

\subsection{Angular resolution of Shashlik + preshower detector}

For the measurement of the angle with the positions of Shashlik and preshower detector, the lever arm $L$ has been estimated from shower simulation. The average electromagnetic shower depth in Shashlik has been evaluated for different energies as the barycenter of the energies in the scintillators,

$$
<l>=\frac{\sum_{i} l_{i} \times E_{i}^{s c i n t i}}{\sum_{i} E_{i}^{s c i n t i}},
$$

where $l_{i}$ is the layer number and $E_{i}^{\text {scinti }}$ is the energy in the $i$-th scintillator. The average layer position is well fitted with,

$$
<l>=9.3+2.2 \times \ln E .
$$

This position does not depend much on the hypothesis assumed for light collection. The radiation length of Shashlik is $16.2 \mathrm{~mm}$, and the shower maximum is at about $7 X_{0}$ ( $=10 X_{0}$ with preshower detector in front) at $80 \mathrm{GeV}$, which is consistent with the value above. The results are summarized in Table 7 and shown in Fig. 21. The angular resolution is well described by $\sigma_{\theta}=70 \mathrm{mrad} / \sqrt{E}$ with $E$ in $\mathrm{GeV}$.

This result is the worst case since the data used are at the tower center as mentioned above. If one analyses the data for the uniformity scan using $80 \mathrm{GeV}$ electrons for two Shashlik towers (one tower plus two half-towers), one obtains the dispersion on $\Delta X$ of $0.97 \mathrm{~mm}$ (it was $1.12 \mathrm{~mm}$ at tower center) which corresponds to $6.8 \mathrm{mrad}$ angular resolution $(\sim 60 \mathrm{mrad} / \sqrt{E})$.

\section{Conclusion}

Newly constructed Shashlik towers were tested with electron beams in the SPS$\mathrm{H} 4$ beam line at CERN. The energy resolution of the bare Shashlik averaged over 4 towers was measured to be $\sigma_{E} / E=8.1 \% / \sqrt{E} \oplus 0.330 / E \oplus 0.5 \%$. With the preshower detector in front, only a slight deterioration of the stochastic term has been observed as, $\sigma_{E} / E=8.7 \% / \sqrt{E} \oplus 0.330 / E \oplus 0.5 \%$. The angular resolution was measured to be better than $70 \mathrm{mrad} / \sqrt{E}$. These measurements satisfy the basic requirements for a sampling electromagnetic calorimeter at the LHC.

\section{Acknowledgements}

We would like to thank the CERN-SPS machine crew and N. Doble for the operation of the beam. We are indebted to M. Haguenauer for his work and organization for data 
taking in the SPS-H4 beam line. We are also grateful to F. Rondeau and J. L. Faure from Dapnia (CEA, Saclay) for their help in tower construction and for the preparation of the mechanical setup.

\section{References}

[1] CMS Collaboration, Letter of Intent for a General Purpose Detector at the LHC, CERN/LHCC 92-3/I1 (1992).

[2] CMS Collaboration, Technical Proposal, CERN/LHCC 94-38 (1994).

[3] CERN-DRDC Proposal-50, "A combined Shashlik + Preshower detector for LHC", J. Badier et al., CERN/DRDC 93-28 (1993).

[4] CERN-DRDC 94-47, RD36 Status Report, Jan. 1995.

[5] H. Fessler et al., Nucl. Instr. and Meth., A228 (1985) 303, ibid. A240 (1985) 284.

[6] G.S. Atoyan et al., Nucl. Instr. and Meth., A320 (1992) 144.

[7] B. Loehr et al., Nucl. Instr. and Meth., A254 (1987) 26.

[8] A. Maio et al., "STIC, The New DELPHI Luminosity Monitor", in Proceeding of the IV International Conference on Calorimetry in High Energy Physics, p.165, Isola d'Elba, Italy, September 19-25, 1993.

[9] W. Brower et al., "Test of a High Resolution Electromagnetic Calorimeter of Wavelength Shifting Fiber Readout", in Proceeding of the V International Conference on Calorimetry in High Energy Physics, BNL, September 25 - October 1, 1994.

[10] T. Lohse et al., "HERA-B Proposal: An Experiment to study CP violation in the B-system using an internal target at the HERA proton ring.", DESY PRC/93-04 (1993), DESY Internal report F15-94-01 (1994).

[11] E. Kistenev et al., in Proceeding of the V International Conference on Calorimetry in High Energy Physics, BNL, September 25 - October 1, 1994.

[12] J. Badier et al., "A Lead Scintillator Electromagnetic Calorimeter for CMS", CMS TN/92-45, X-LPNHE/93-02 (1992).

[13] J. Badier et al., "Position and Angular Resolution with the Shashlik Calorimeter", CMS TN/93-65, X-LPNHE/93-03 (1993).

[14] L. Dobrzynski, "Shashlik calorimeter. Response to high energy electrons", Nucl. Instr. and Meth., A344 (1994) 57.

[15] J. Badier et al., "Shashlik calorimeter. Beam test results", Nucl. Instr. and Meth., A348 (1994) 74.

[16] J. Badier et al., "New Test Beam Results of Shashlik and Preshower Prototypes", CMS TN/94-152, INR-984/94, X-LPNHE/94-01 (1994).

[17] "Shashlik Calorimeter for CMS", in Proceeding of the IV International Conference on Calorimetry in High Energy Physics, p.158, Isola d'Elba, Italy, September 19-25, 1993.

[18] "Shashlik Calorimetry : an Electromagnetic Calorimeter + Preshower for LHC", talk presented at the $\mathrm{V}$ International Conference on Calorimetry in High Energy Physics, BNL, USA, September 25 - October 1st, 1994.

[19] P. Aspell et al., "Beam Test Results of a Shashlik Calorimeter in High Magnetic Field", CERN-PPE/95-152 (1995), to be published in NIM.

[20] E. Beuville et al., Nucl. Instr. and Meth., A288 (1990) 157, Nucl. Phys. B (Proc. Suppl.), 23A (1991) 198, IEEE Trans. Nucl. Sci. 39 (1992) 766.

[21] J. Buskeus et al., NIM 207 (1983) 365.

[22] R. Wigmans, Nucl. Instr. and Meth., A259 (1987) 389. 


\section{Shashlik Testbeam Setup}

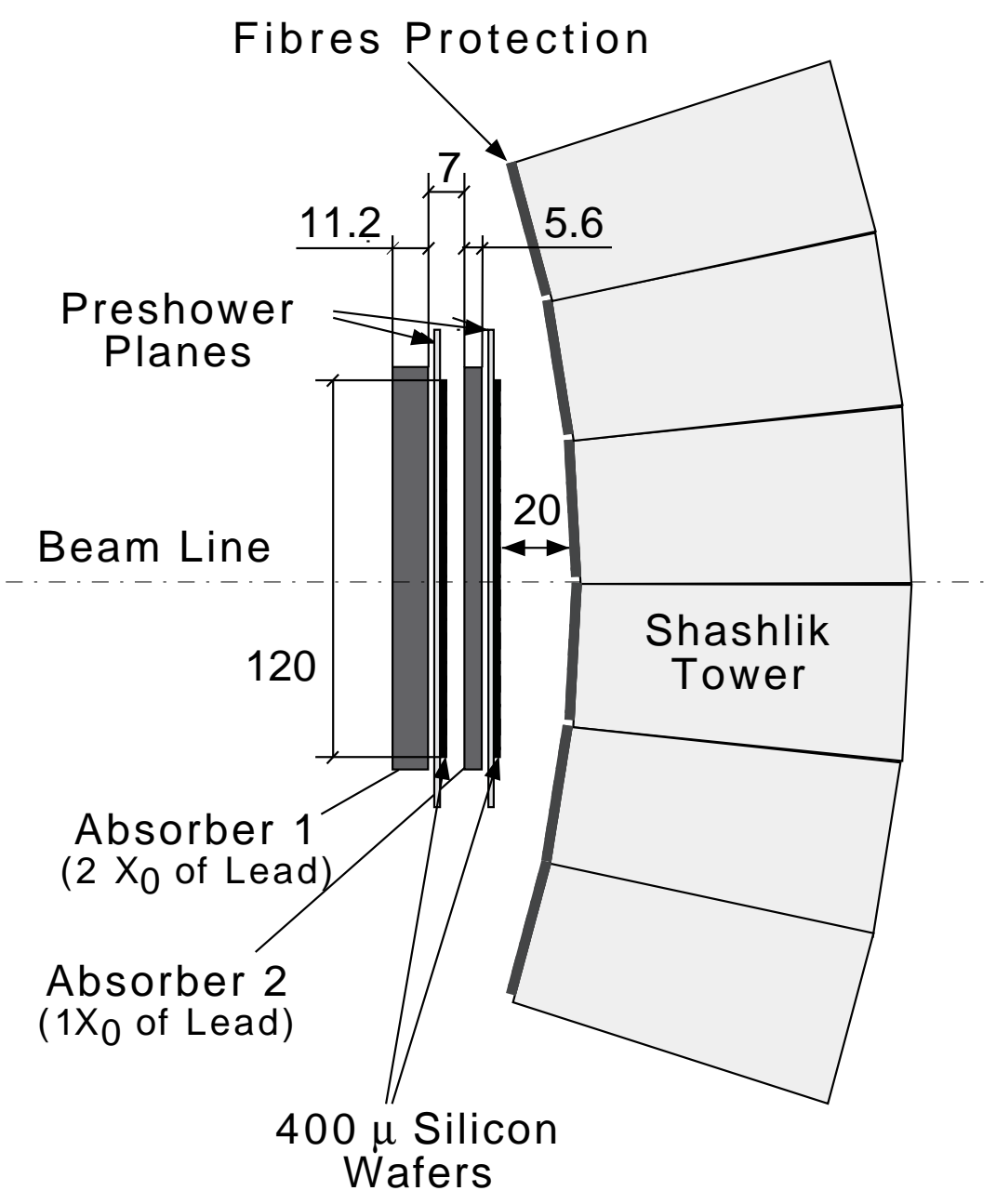

Figure 1: Detector geometry for H4 beam test in 1994. 


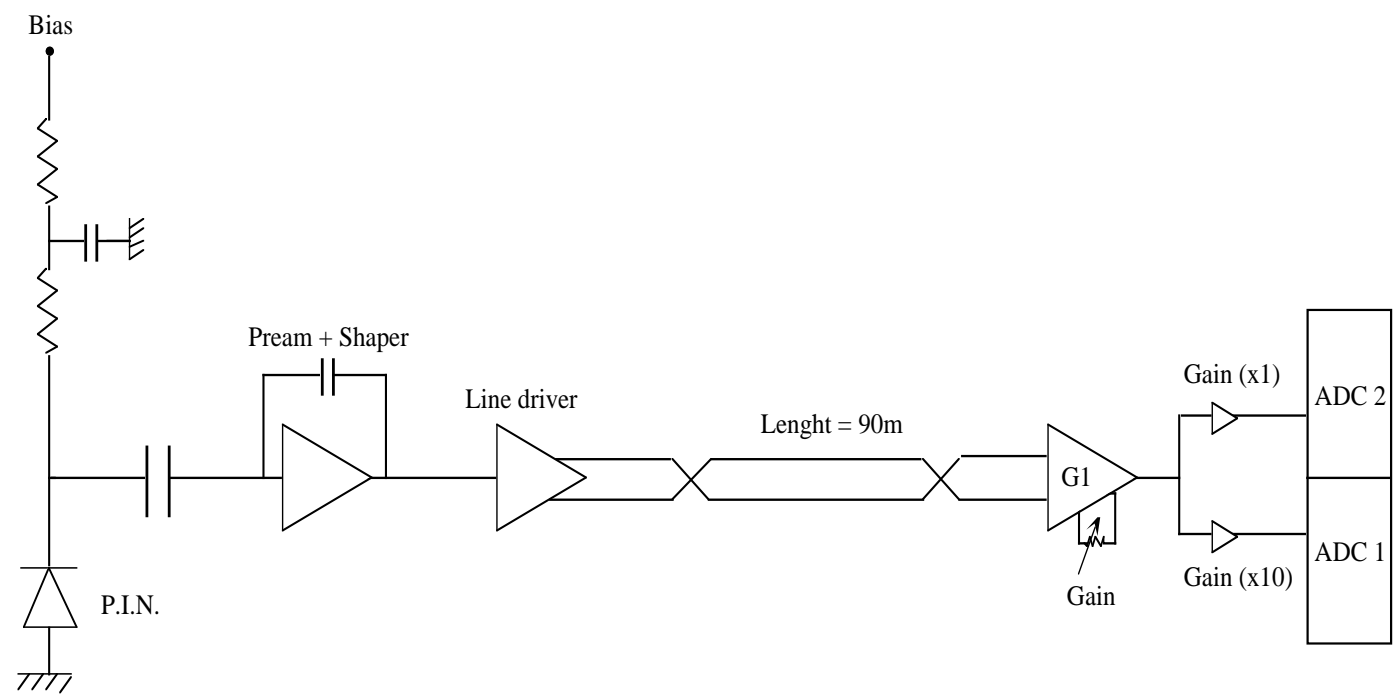

Figure 2: Scheme of the readout electronics for beam test. 


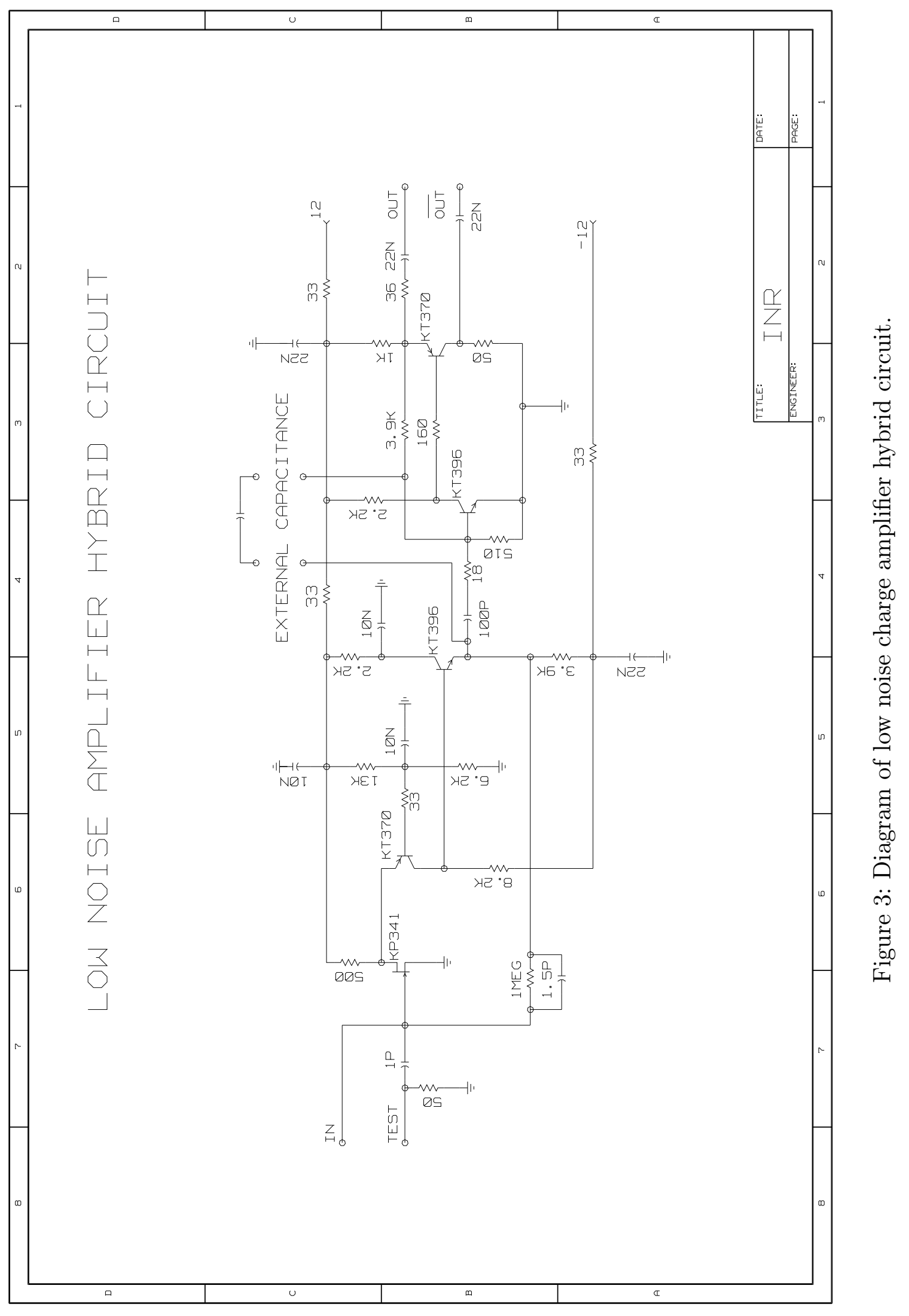



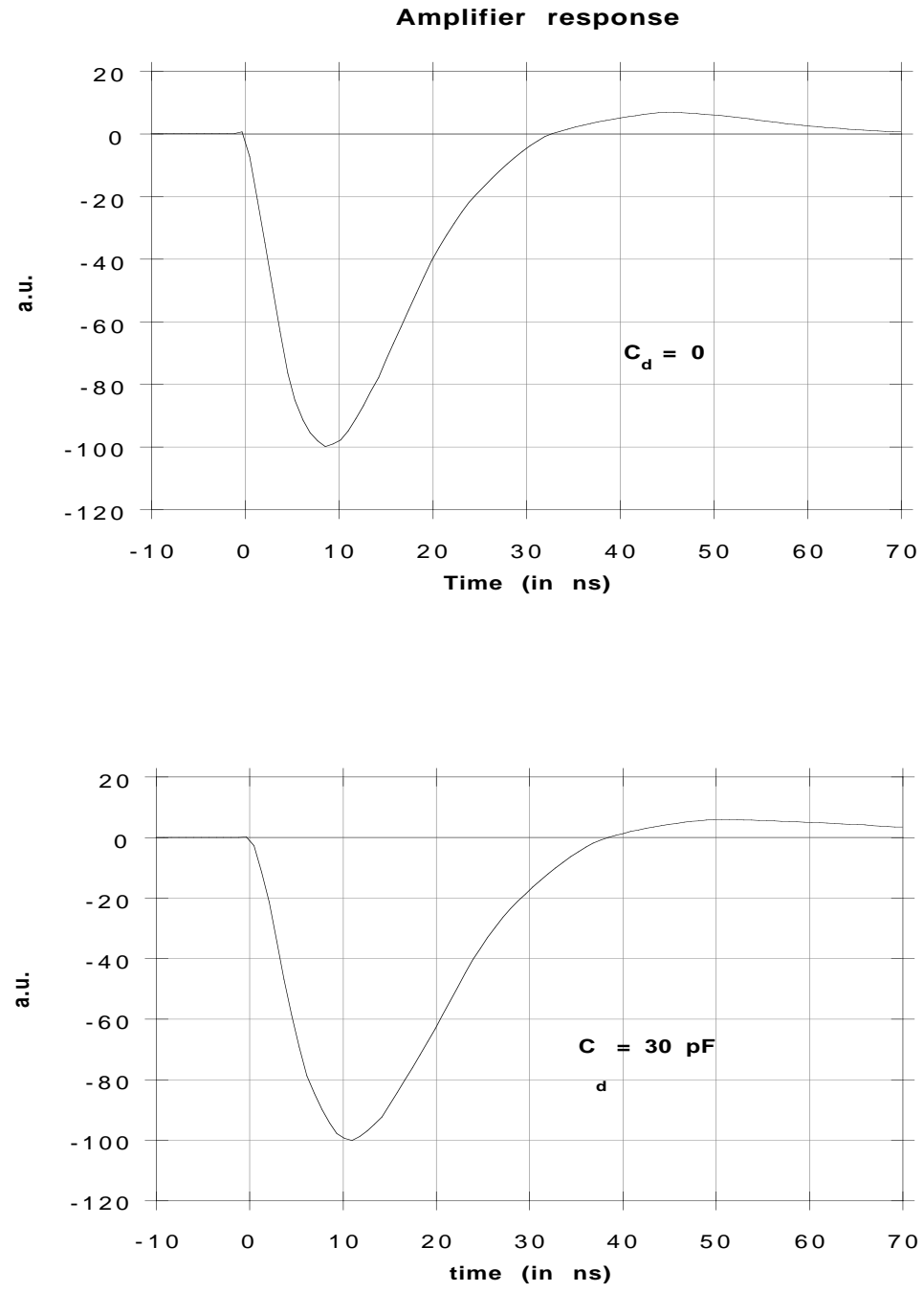

Figure 4: Current response of the hybrid amplifier for two different detector capacitance (0 and $30 \mathrm{pF}$ ). 


\section{ENC vs Capacitance}

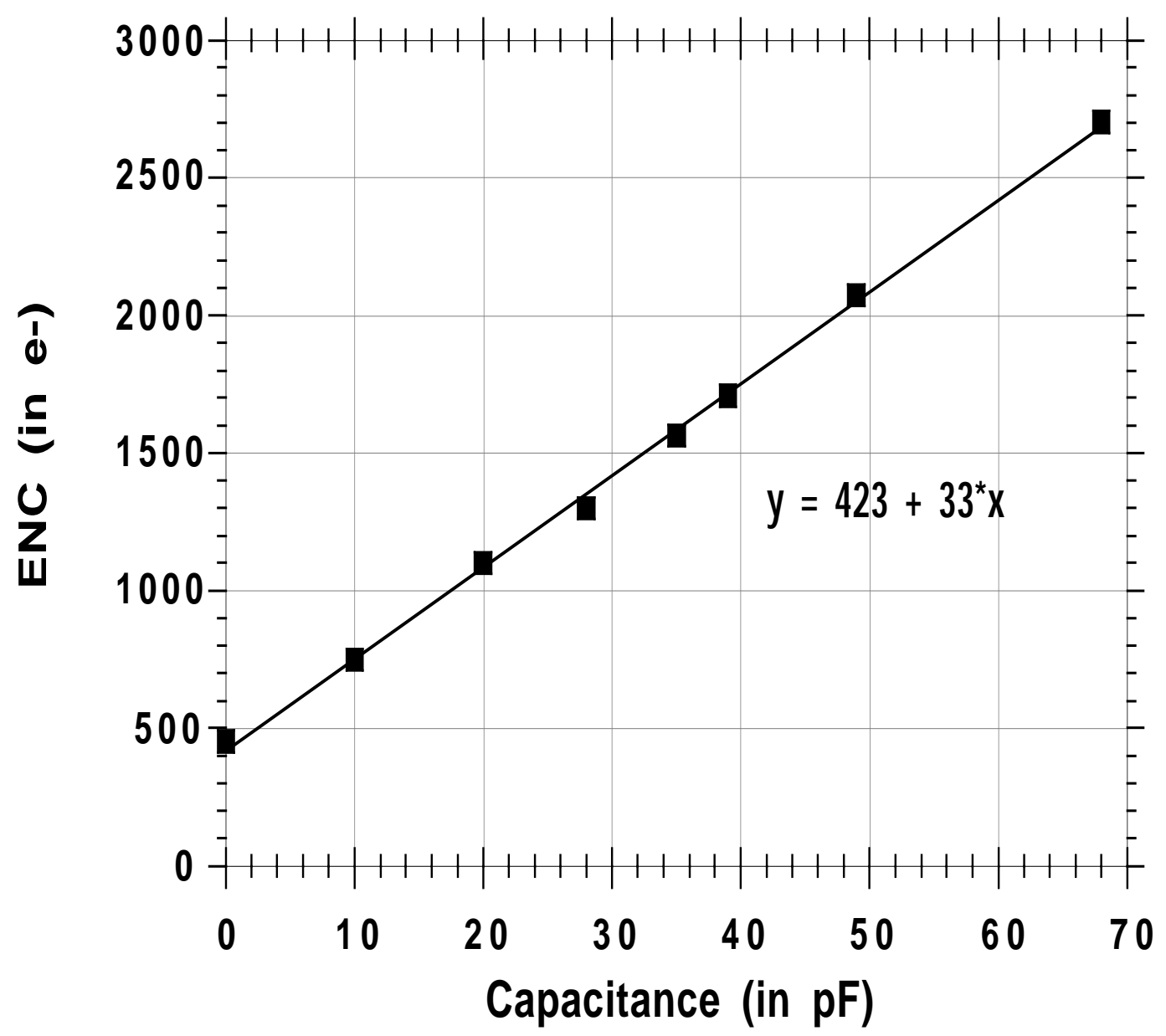

Figure 5: ENC of the hybrid amplifier as function of the detector capacitance. 

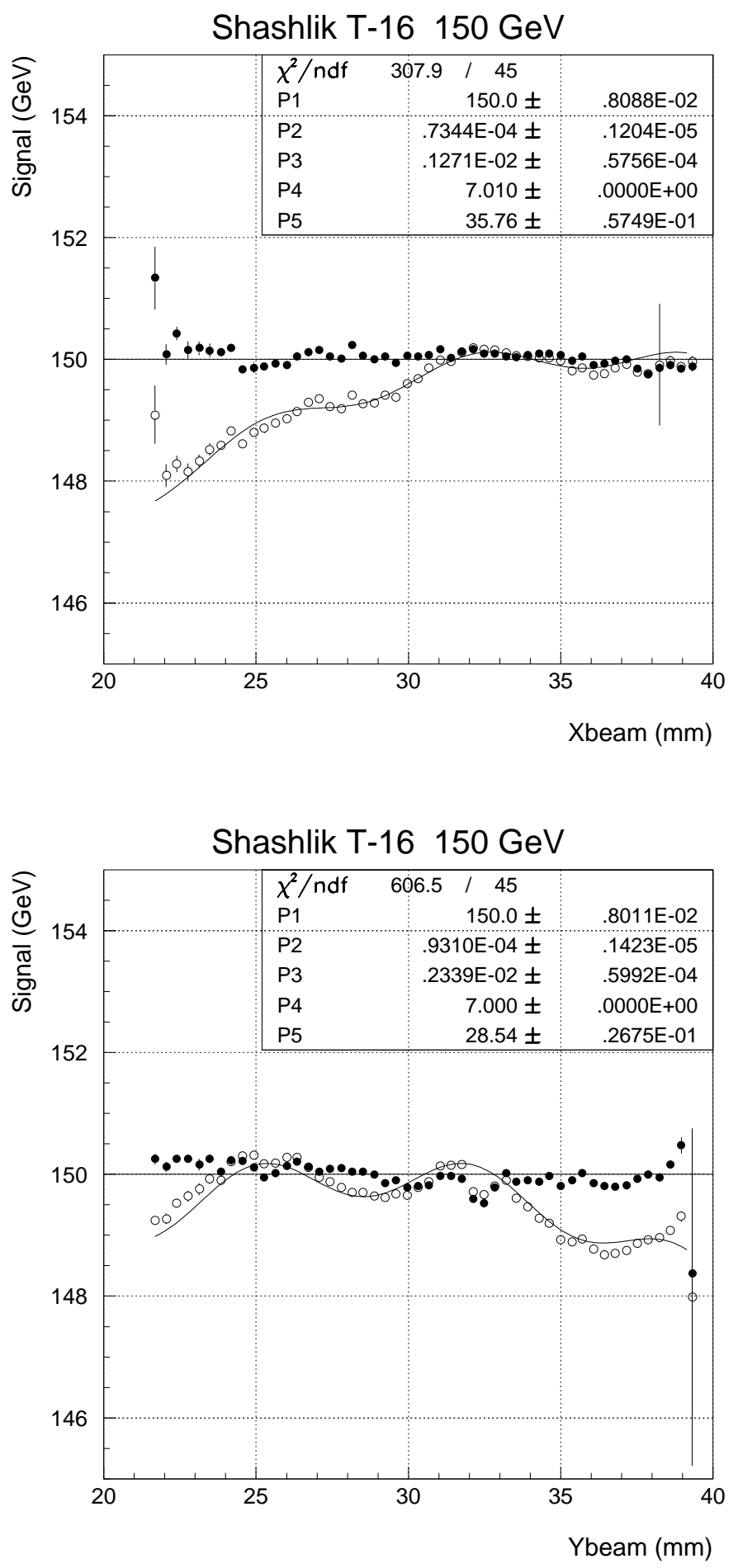

Figure 6: Lateral non-uniformity correction for Shashlik response for $150 \mathrm{GeV}$ electrons. The signals are plotted as a function of beam impact point $x$ (horizontal, tilted 3 degree) and $y$ (vertical, non-tilted) for before correction (open circle) and after correction (closed circle). The curves are the results of the fit. 


\section{Shashlik Projective - Linearity}

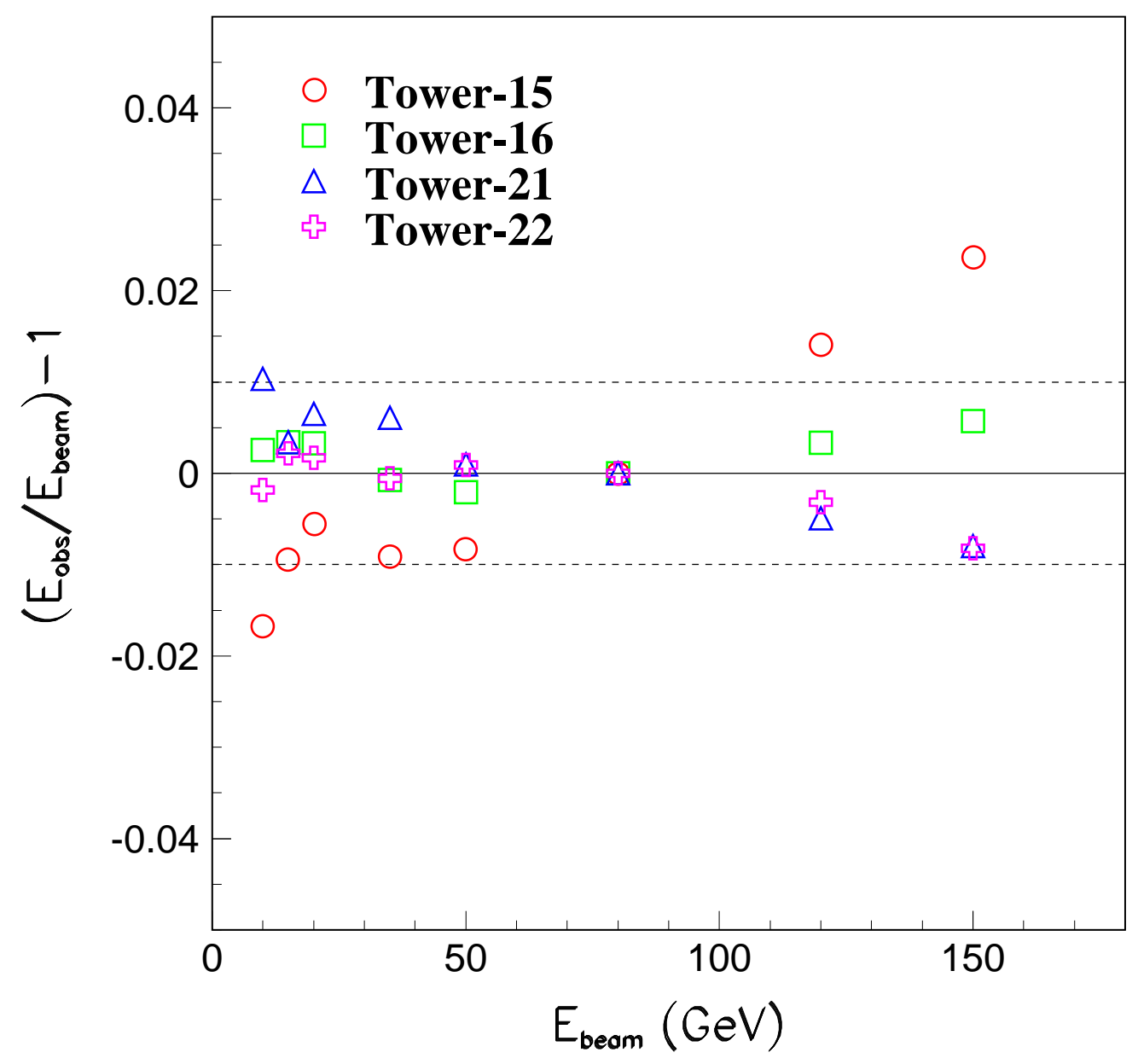

Figure 7: Shashlik signal linearity for data at $\mathrm{H} 4$ beam line. Data are normalized at $80 \mathrm{GeV}$. 


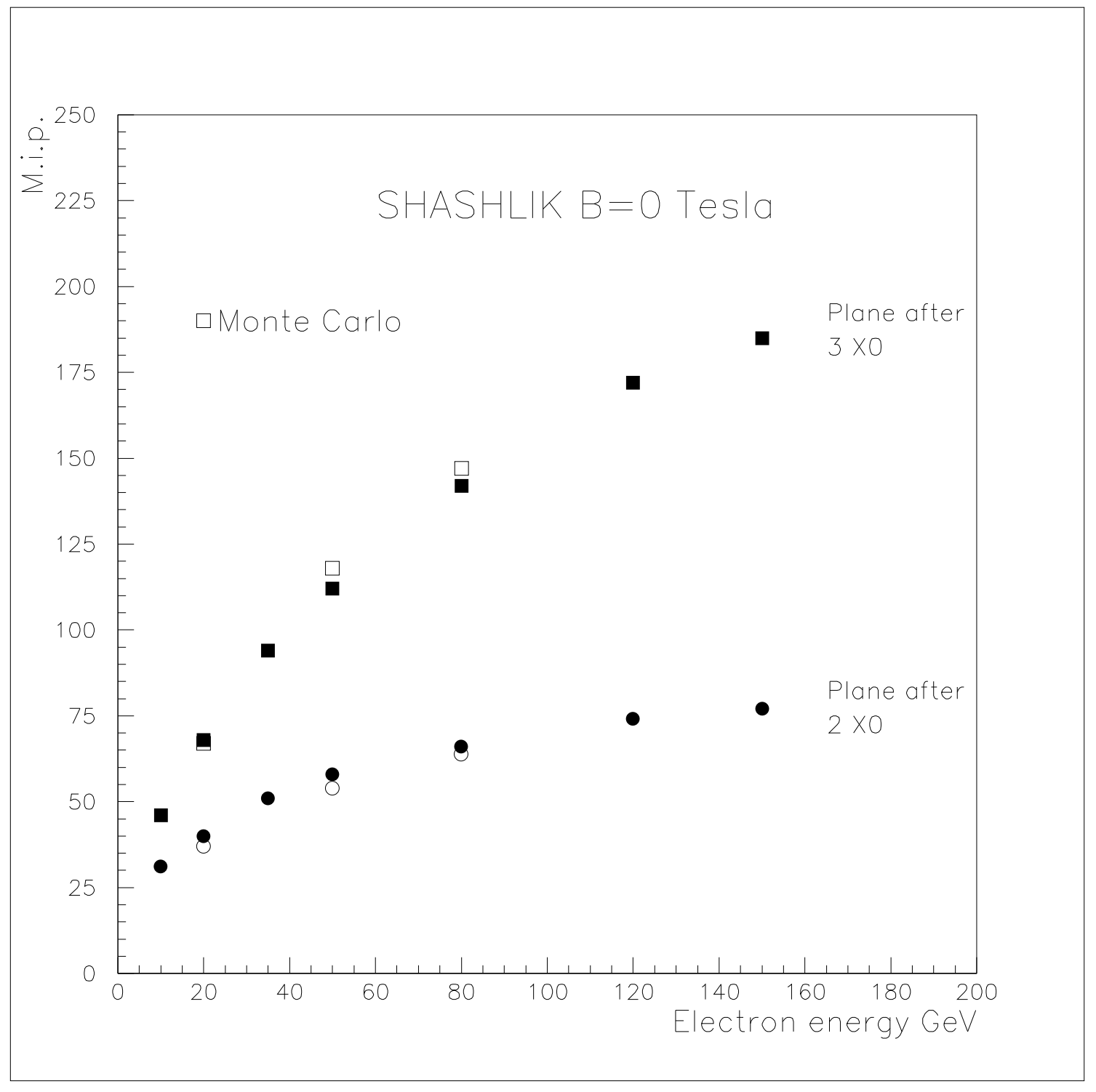

Figure 8: Preshower detector average signal for electrons in the first layer (after $2 X_{0}$ ) and in the second layer (after $3 X_{0}$ ). 


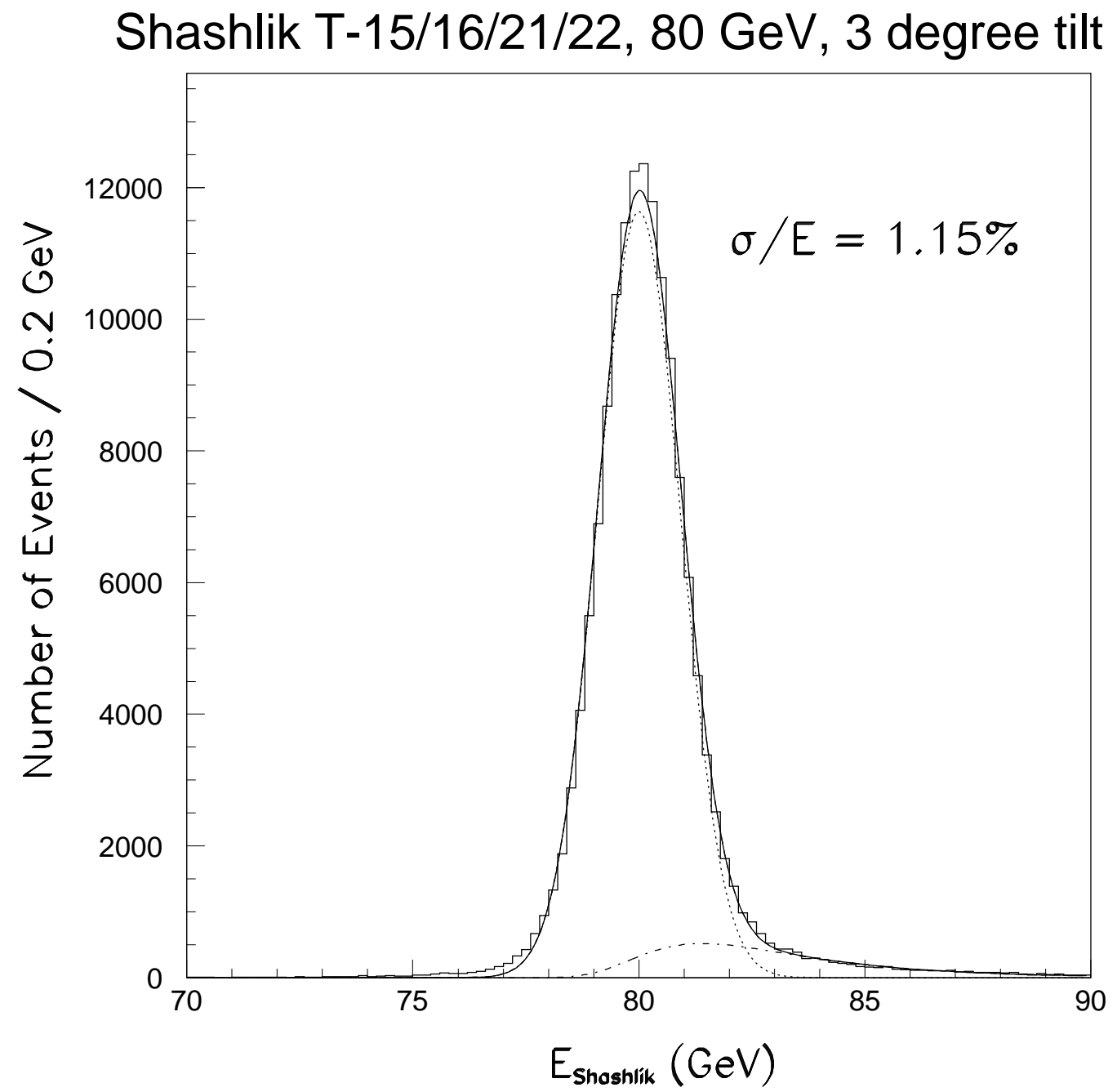

Figure 9: Reconstructed energy in the nine towers centred on Towers 15, 16, 21 and 22 for electrons of $80 \mathrm{GeV}\left(2 \times 2 \mathrm{~cm}^{2}\right)$. The solid curve is the result of fit with Gaussian (dotted line) plus Landau (dot-dashed line) distribution. The Landau tail is to account for the nuclear counter effect. 


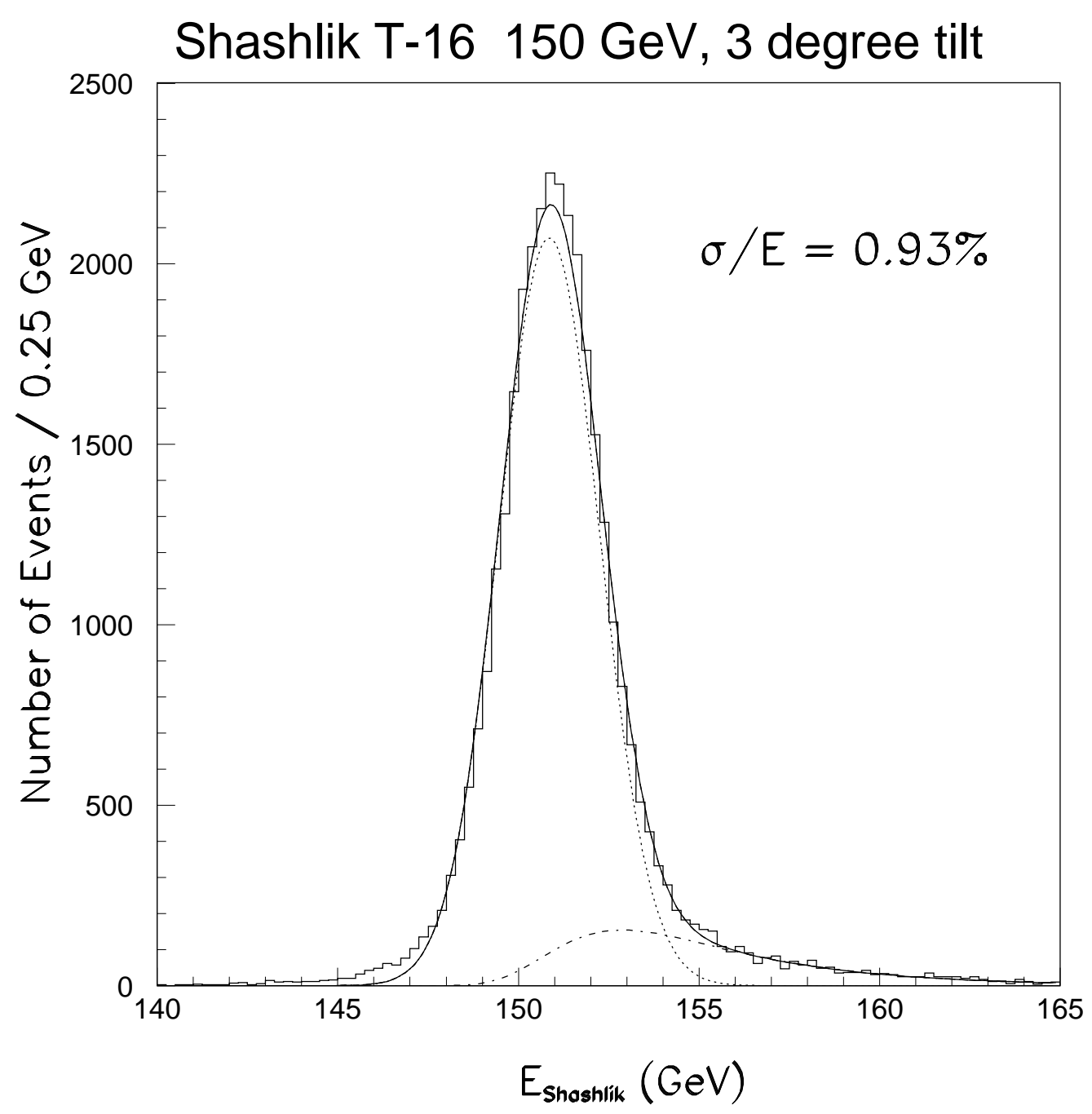

Figure 10: Reconstructed energy in the nine towers centred on Tower-16 for electrons of $150 \mathrm{GeV}$. The curves are the same as in the previous figure. The calibration constants are those obtained for $80 \mathrm{GeV}$ data which shift the peak value slightly above $150 \mathrm{GeV}$ due to non-linearity. 


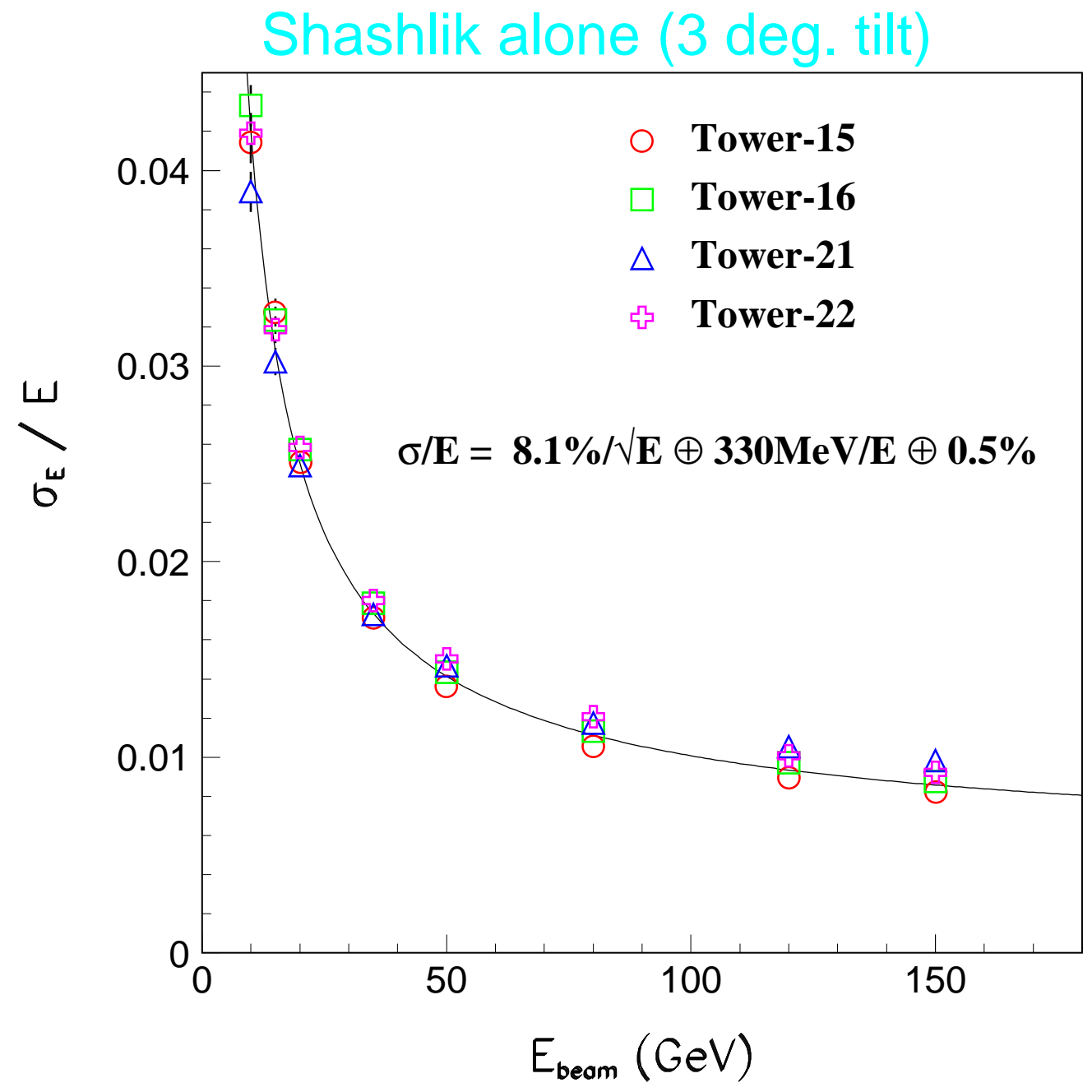

Figure 11: Energy resolution of bare Shashlik prototype as a function of the electron beam energy. 


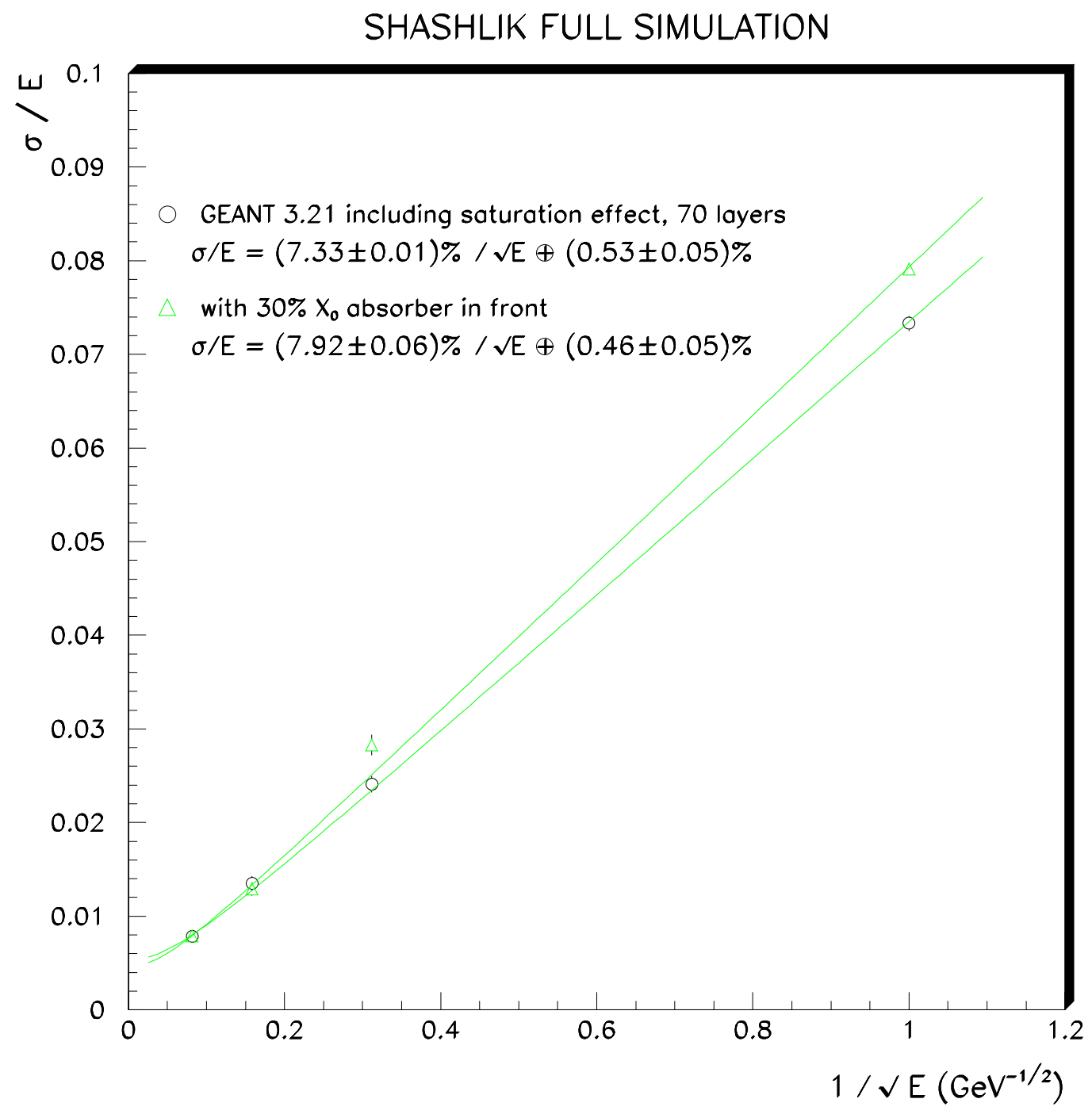

Figure 12: Monte Carlo simulation on the effect of absorber material in front of Shashlik. The total tower radiation length is $25.7 X_{0}$. It is assumed that the calorimeter is laterally infinite. The curves are the results of the fit. 


\section{$\mathrm{e}^{-}, 200 \mathrm{GeV}$, SHOWER LEAKAGE}

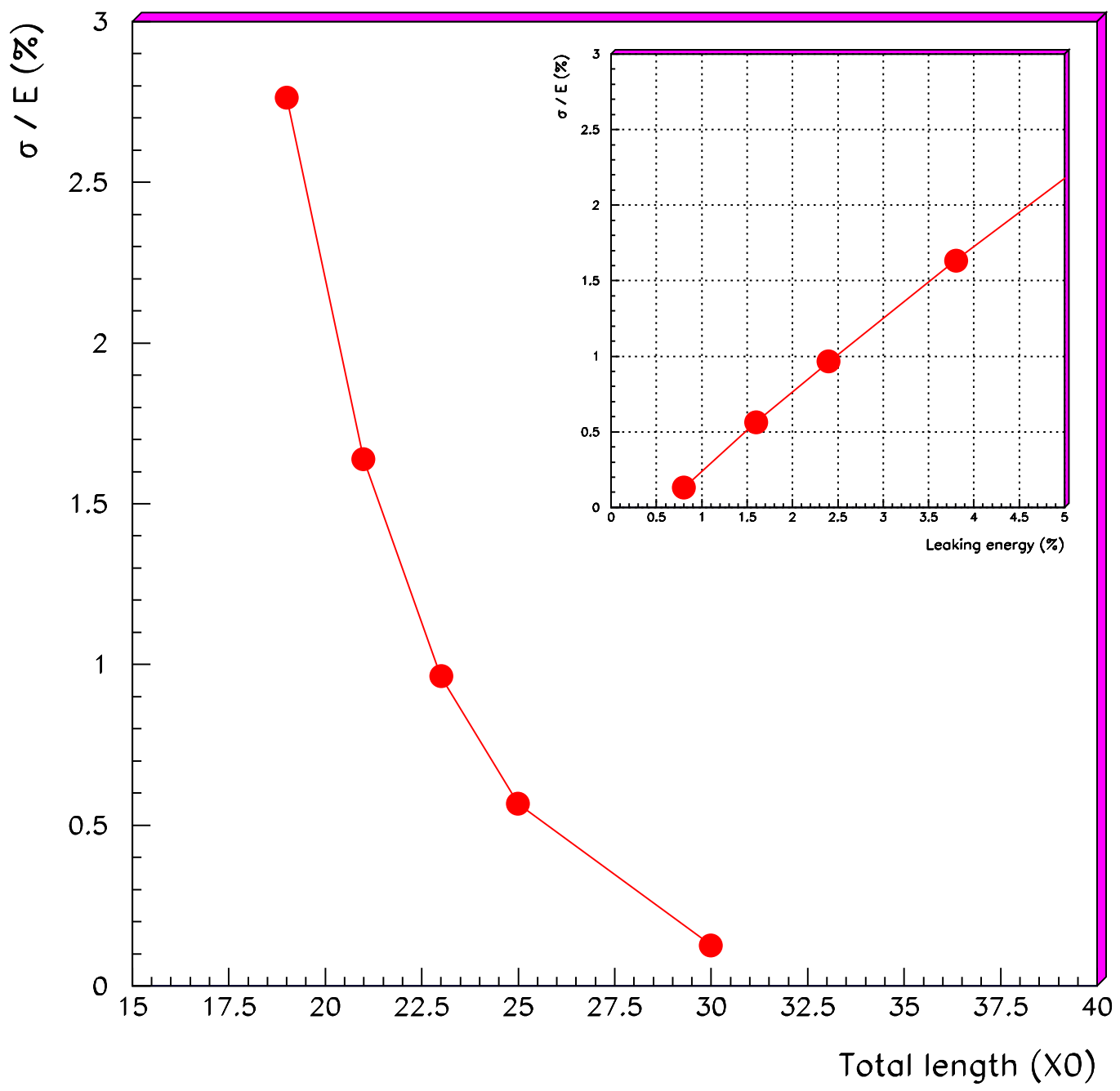

Figure 13: Monte Carlo simulation on the effect of shower leakage. The geometry is the same as in the previous figure. The contribution to the energy resolution for $200 \mathrm{GeV}$ electrons is plotted as functions of the total thickness of the calorimeter and of the leaking energy. 


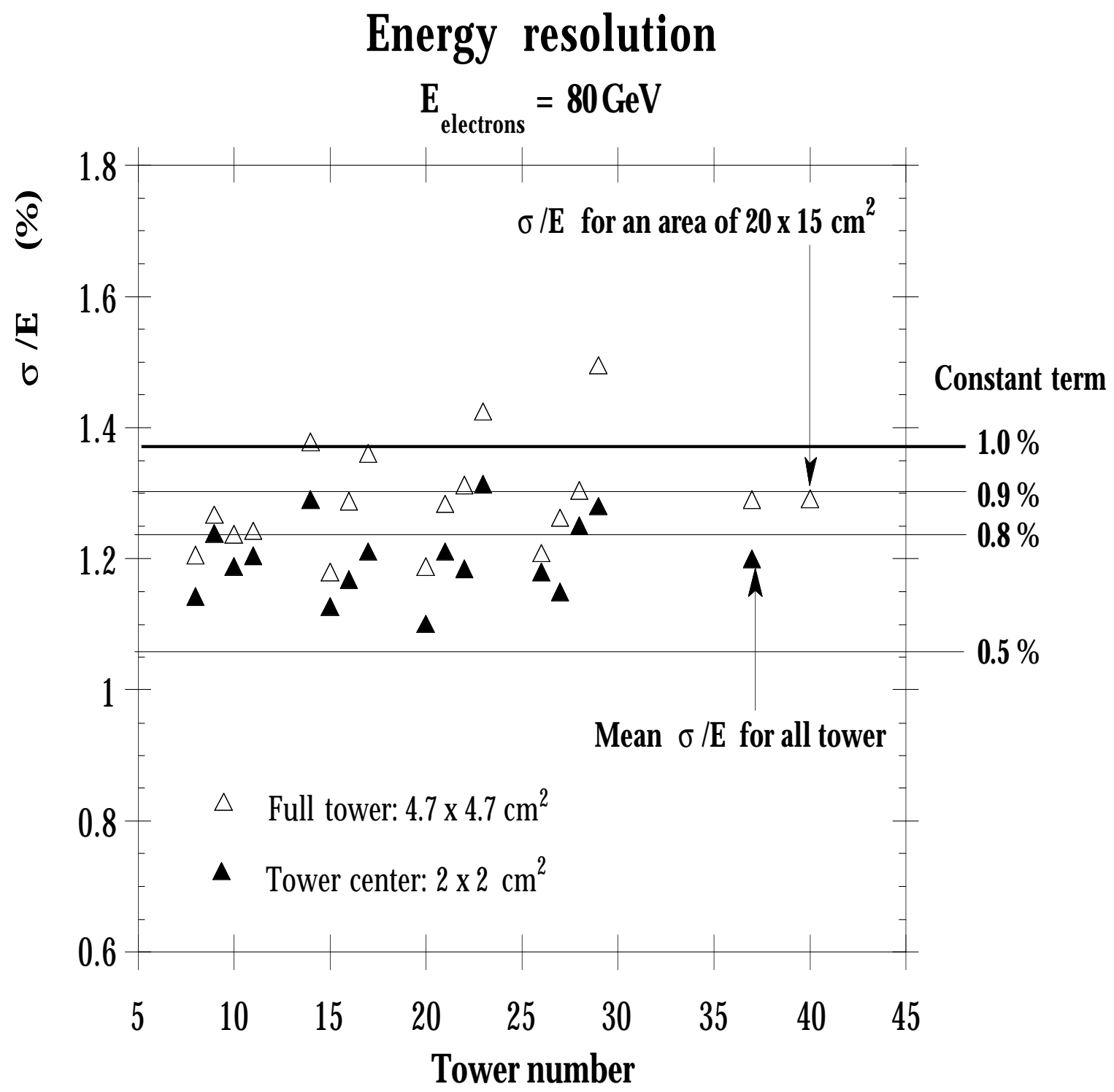

Figure 14: Energy resolution for 16 Shashlik towers after uniformity correction at tower center $\left(2 \times 2 \mathrm{~cm}^{2}\right.$ area $)$ and for the full towers $\left(4.7 \times 4.7 \mathrm{~cm}^{2}\right.$ area). The mean values are also plotted. The horizontal lines give the expectations for various values of constant term, whereas the stochastic term is fixed to $8.1 \%$. 


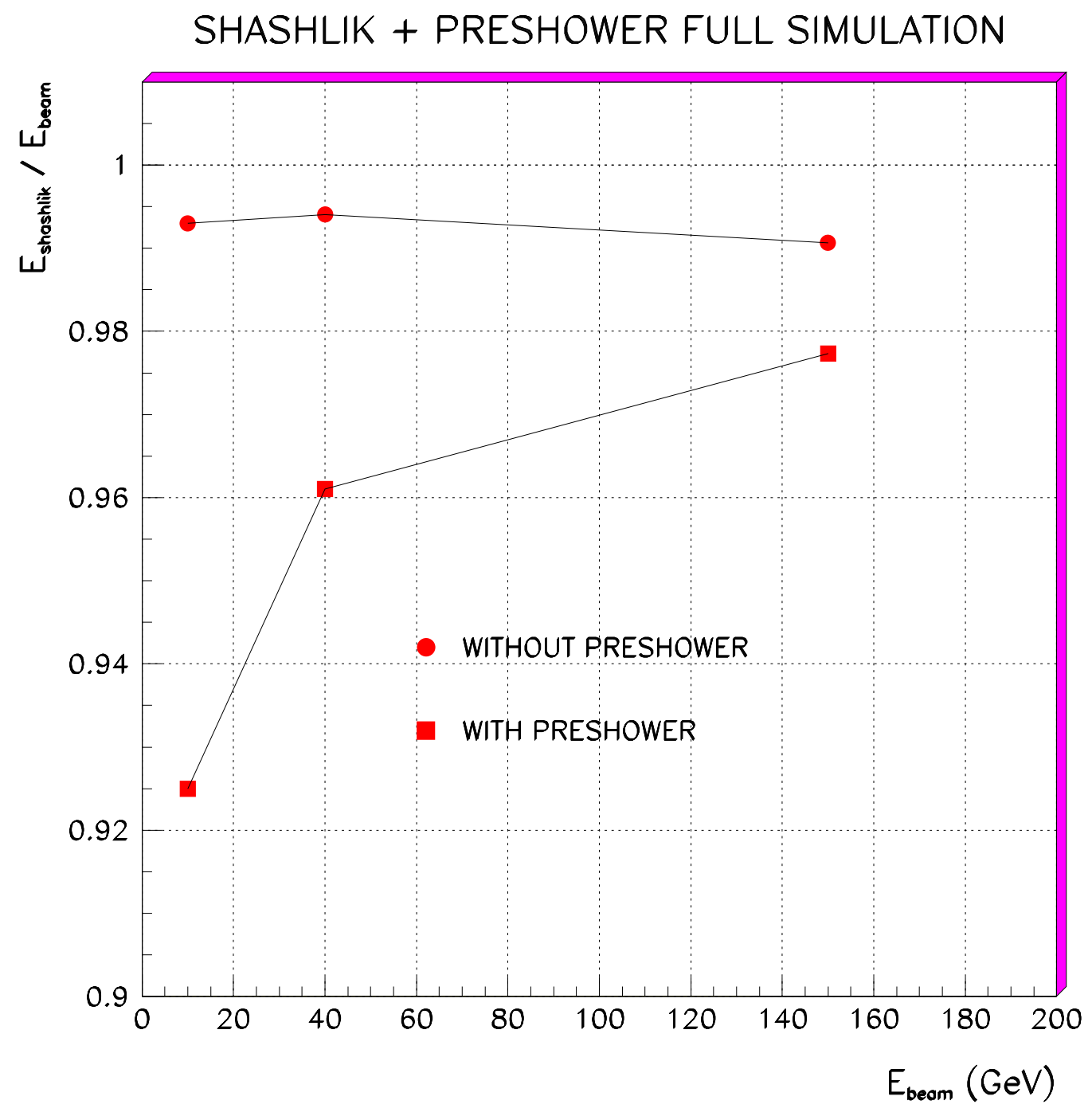

Figure 15: Monte Carlo simulation on the effect of preshower detector. The average Shashlik energy (normalized to total incident energy) are plotted for 10,40 and $150 \mathrm{GeV}$ electrons. The geometry is exactly the same as SPS-H4 beam line setup. 


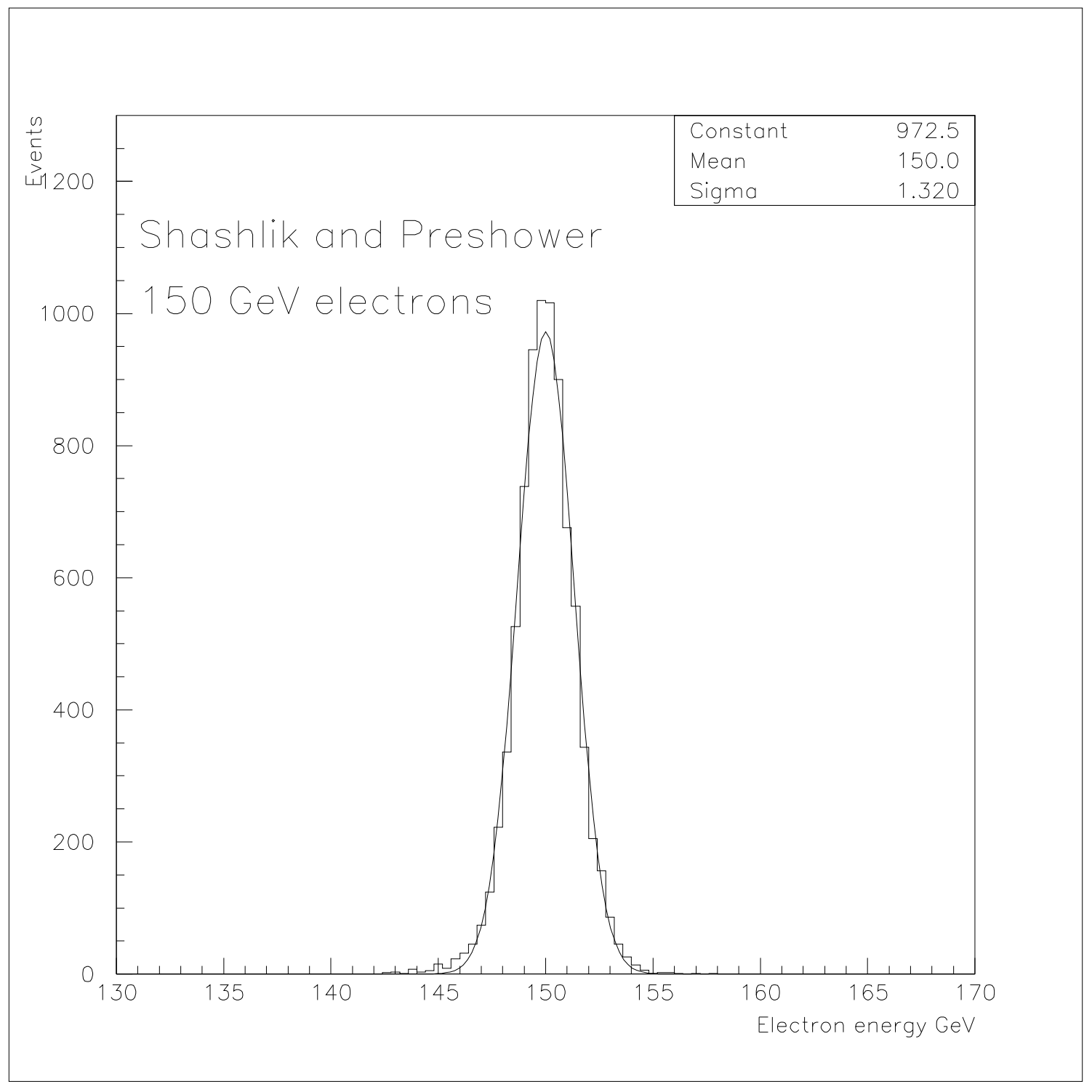

Figure 16: Reconstructed energy in Shashlik and preshower detector for electron $150 \mathrm{GeV}$ for Tower-15. The solid curve is the result of the fit with Gaussian function. 


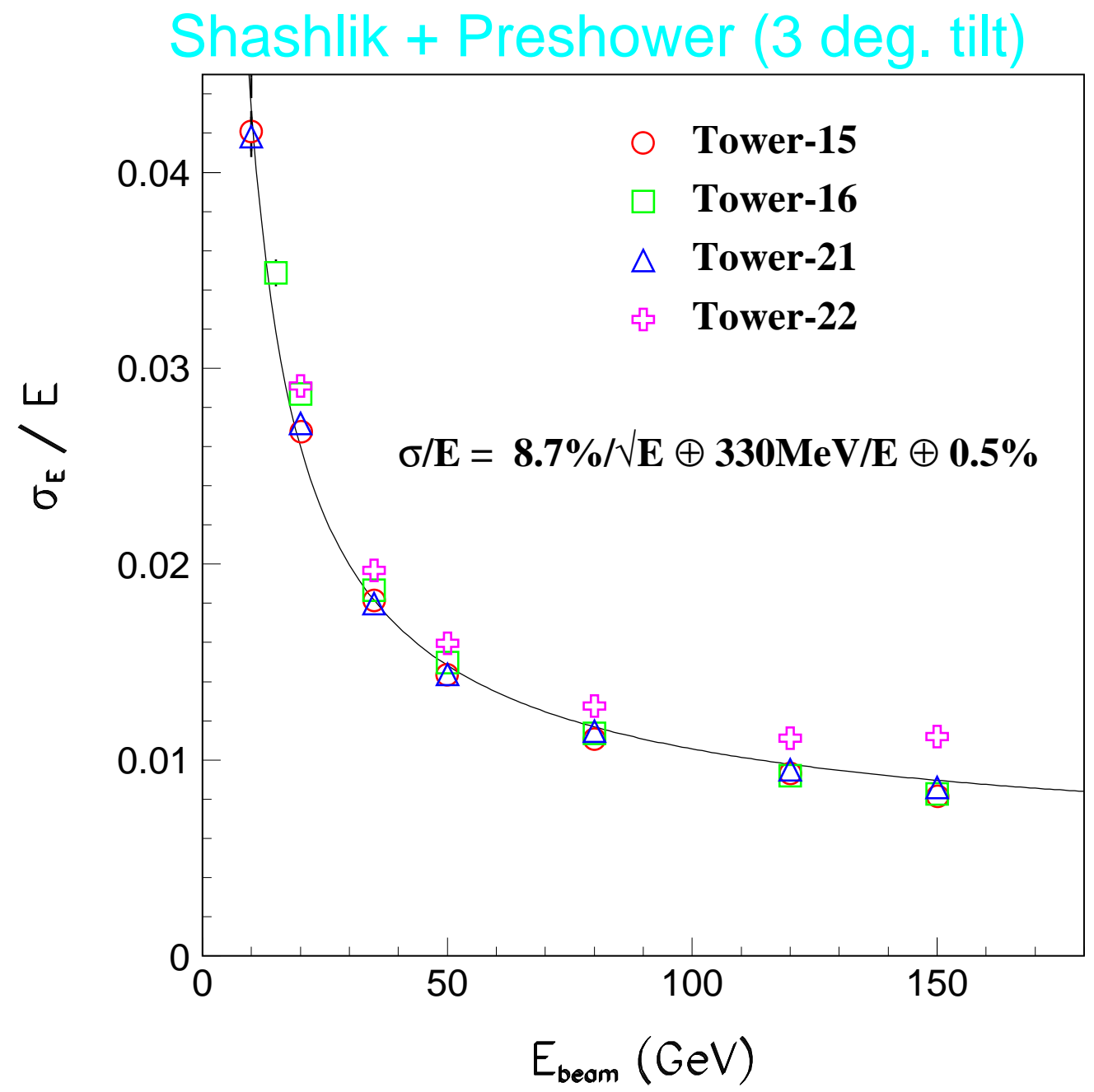

Figure 17: Energy resolution of Shashlik plus preshower detector as a function of the electron beam energy. 


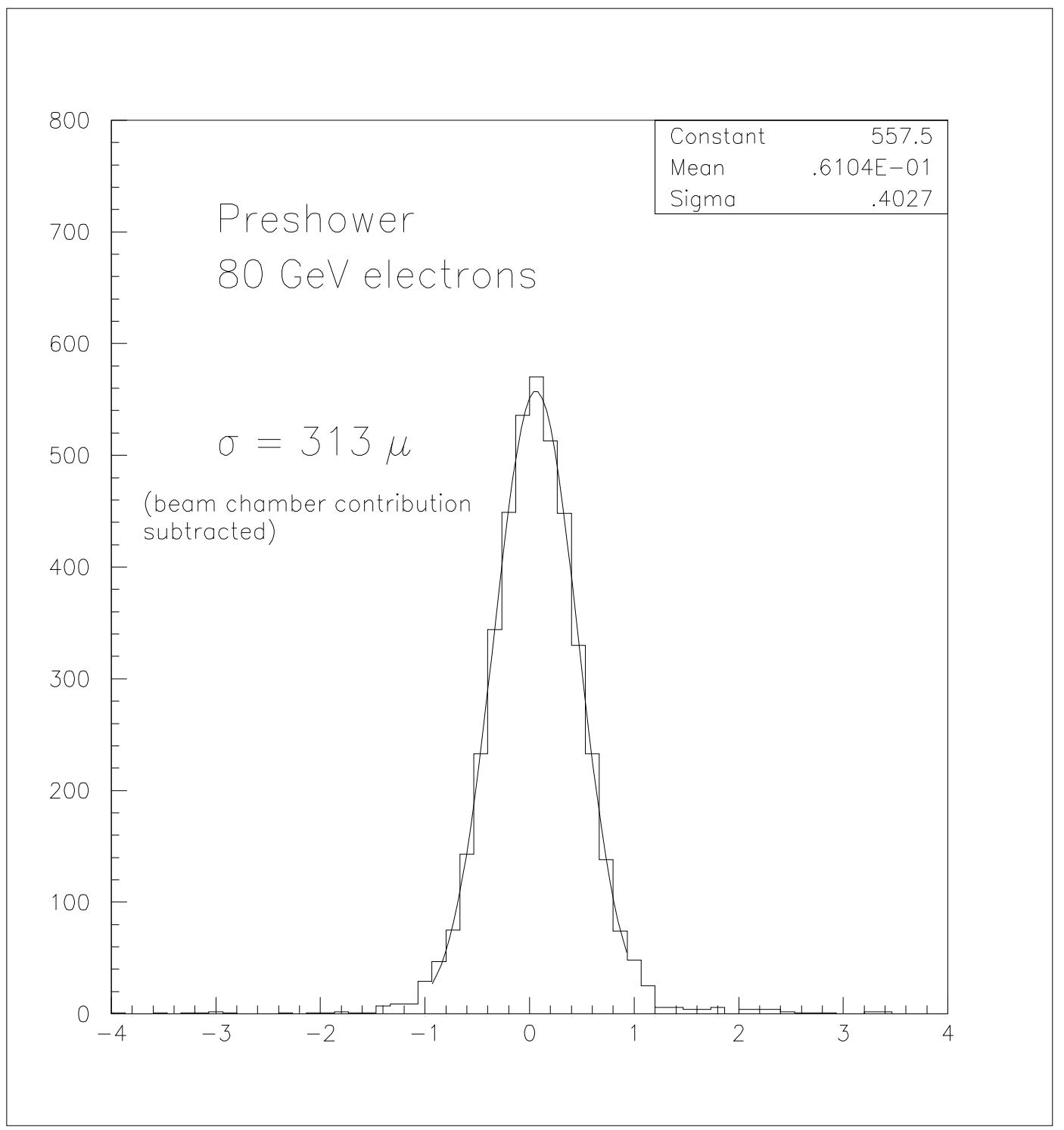

Figure 18: Preshower detector position resolution for $80 \mathrm{GeV}$ electrons. 


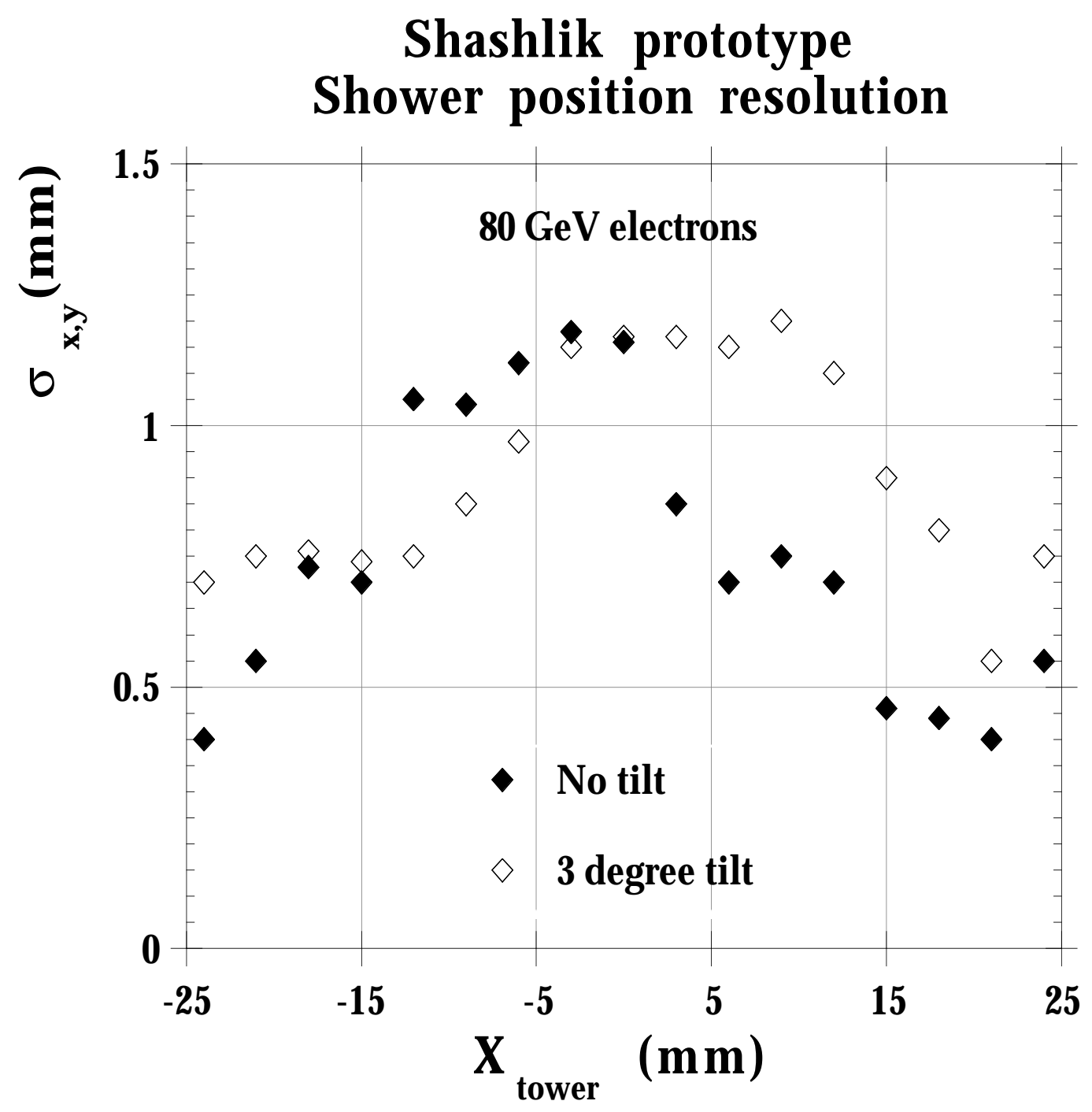

Figure 19: Shashlik position resolution for the non-tilt and the 3 degree tilted configurations as a function of the beam impact point. 


\section{Shashlik position resolution}

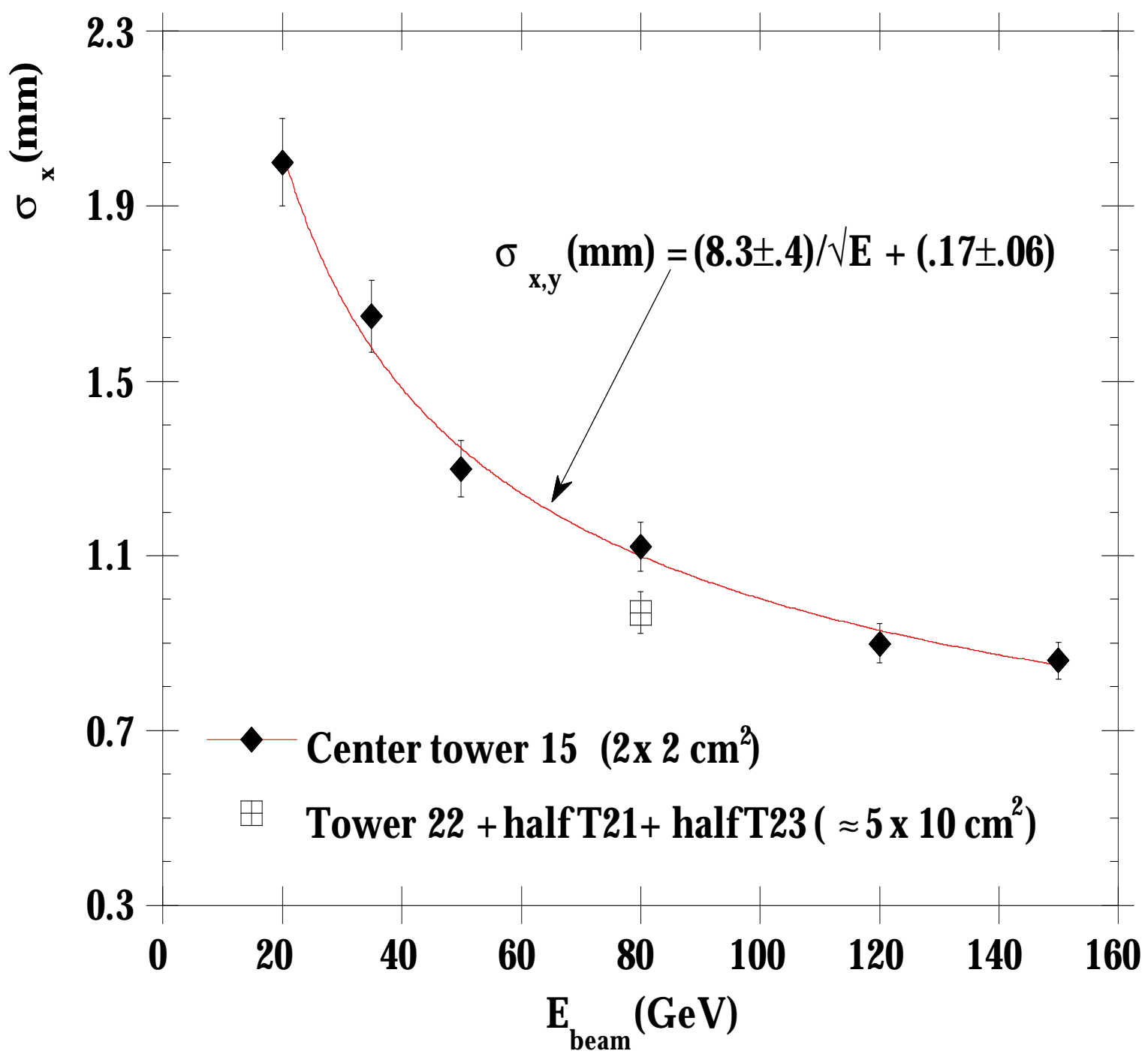

Figure 20: Shashlik position resolution as a function of the energy for Tower-22. The curve is the result of the fit. 


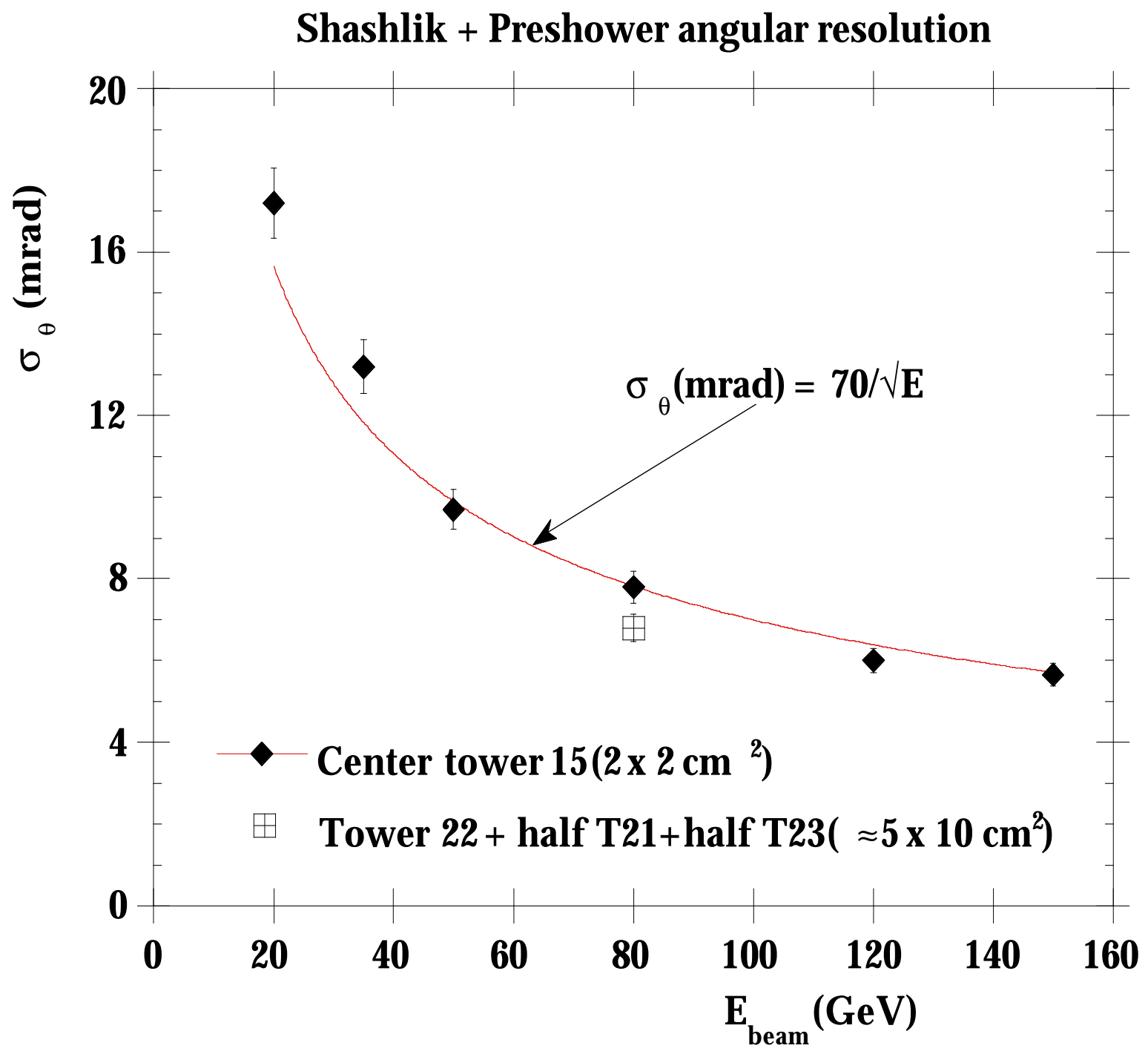

Figure 21: The angular resolution of Shashlik plus preshower system as a function of the beam energy. 